University of South Florida

DIGITAL COMMONS

Digital Commons @ University of

@ UNIVERSITY OF SOUTH FLORIDA

South Florida

$10-31-2008$

\title{
Development and Psychometric Evaluation of the Chemotherapy Induced Peripheral Neuropathy Assessment Tool
}

Cindy S. Tofthagen

University of South Florida

Follow this and additional works at: https://digitalcommons.usf.edu/etd

Part of the American Studies Commons

\section{Scholar Commons Citation}

Tofthagen, Cindy S., "Development and Psychometric Evaluation of the Chemotherapy Induced Peripheral Neuropathy Assessment Tool" (2008). USF Tampa Graduate Theses and Dissertations.

https://digitalcommons.usf.edu/etd/535

This Dissertation is brought to you for free and open access by the USF Graduate Theses and Dissertations at Digital Commons @ University of South Florida. It has been accepted for inclusion in USF Tampa Graduate Theses and Dissertations by an authorized administrator of Digital Commons @ University of South Florida. For more information, please contact digitalcommons@usf.edu. 
Development and Psychometric Evaluation of the Chemotherapy Induced Peripheral Neuropathy Assessment Tool

by

Cindy S. Tofthagen

A dissertation submitted in partial fulfillment

of the requirements for the degree of

Doctor of Philosophy

College of Nursing

University of South Florida

\author{
Major Professor: Susan C. McMillan, Ph.D., A.R.N.P. \\ Jason Beckstead, Ph.D. \\ Lois Gonzalez, Ph.D., A.R.N.P. \\ Janine Overcash, Ph.D., A.R.N.P.
}

Date of Approval:

October 31, 2008

Keywords: oncology, cancer, performance, symptom, nursing

(C) Copyright 2008, Cindy Tofthagen 
Table of Contents

List of Tables $\quad$ iv

List of Figures $\quad$ V

Abstract vi vi

Chapter One: Introduction 1

Underlying mechanisms $\quad 2$

Risk factors and symptoms $\quad 2$

Definition of terms $\quad 4$

Chemotherapy induced peripheral neuropathy 4

Painful neuropathy 4

Non-painful neuropathy 4

Symptom interference $\quad 5$

Measurement $\quad 5$

Clinical evaluation $\quad 6$

$\begin{array}{ll}\text { Self-report } & 6\end{array}$

$\begin{array}{ll}\text { Statement of the problem and purpose } & 7\end{array}$

$\begin{array}{ll}\text { Conceptual framework } & 8\end{array}$

$\begin{array}{lr}\text { Characteristics } & 9\end{array}$

$\begin{array}{ll}\text { Timing } & 10\end{array}$

$\begin{array}{ll}\text { Intensity } & 10\end{array}$

$\begin{array}{ll}\text { Distress } & 10\end{array}$

Physical performance $\quad 11$

$\begin{array}{ll}\text { Significance to nursing } & 11\end{array}$

Chapter Two: Review of the Literature $\quad 12$

$\begin{array}{lr}\text { Peripheral Neuropathy Scale } & 12\end{array}$

FACT/GOG Neurotoxicity Subscale $\quad 17$

Quality of Life Questionnaire-Chemotherapy Induced
Peripheral Neuropathy 20

$\begin{array}{ll}\text { Summary } & 23\end{array}$

Chapter Three: Phase I Method, Results and Discussion 24

$\begin{array}{ll}\text { Method } & 24\end{array}$

Sample and setting $\quad 24$

$\begin{array}{ll}\text { Procedures } & 25\end{array}$ 
$\begin{array}{ll}\text { Results } & 26\end{array}$

Demographics 26

Borderline case $\quad 27$

$\begin{array}{ll}\text { Non-painful symptoms } & 27\end{array}$

Risk of injury 28

Painful symptoms $\quad 29$

Interference with activities $\quad 31$

Instrument development $\quad 32$

Painful symptoms $\quad 33$

Non-painful symptoms $\quad 34$

Symptom Interference $\quad 34$

Discussion

Chapter Four: Phase II Method, Results and Discussion 38

$\begin{array}{ll}\text { Method } & 38\end{array}$

Consultants $\quad 38$

Procedures $\quad 40$

Results 41

Scoring $\quad 41$

Discussion $\quad 42$

Chapter Five: Phase III Method, Results and Discussion 44

Method 44

Instruments $\quad 45$

Functional Assessment of Cancer Therapy 45

Neurotoxicity Subscale

Chemotherapy Induced Peripheral 45

Neuropathy Assessment Tool

Demographic Data Form 46

$\begin{array}{ll}\text { Procedures } & 46\end{array}$

Evaluating Validity $\quad 48$

Evaluating Reliability $\quad 50$

Data Analysis $\quad 50$

Results $\quad 51$

Demographics $\quad 51$

Descriptive symptom management $\quad 52$

Validity

54

Reliability $\quad 58$

Discussion $\quad 59$

Validity $\quad 59$

Reliability $\quad 60$

Revisions to the CIPNAT 60

Scoring of the revised CIPNAT $\quad 61$

$\begin{array}{ll}\text { Limitations } & 62\end{array}$

Implications for Nursing $\quad 63$ 
Directions for Future Research $\quad 64$

Conclusions 64

$\begin{array}{ll}\text { References } & 65\end{array}$

$\begin{array}{ll}\text { Appendices } & 69\end{array}$

$\begin{array}{ll}\text { Appendix A: FACT/GOG-Ntx Version } 4 & 70\end{array}$

Appendix B: CIPNAT $\quad 71$

Appendix C: Demographic Data Form $\quad 82$

Appendix D: Moffitt SRC Approval Letter $\quad 84$

Appendix E: Letter of approval, physician's office 86

Appendix F: CIPNAT (Version 1) 87

About the Author $\quad$ End Page 


\section{List of Tables}

Table 1. Frequencies and percentages for race, gender, cancer type and neurotoxic agent

Table 2. Frequencies and percentages for non-painful symptoms

Table 3. Frequencies and percentages for painful symptoms

Table 4. Frequencies and percentages for interference with usual activities

Table 5. Scoring guide

Table 6. Frequencies and percentages of demographic data

Table 7. Occurrence, severity, intensity, distress and timing of neuropathic symptoms

Table 8. Frequency of interference items

Table 9. T-tests for CIPNAT and FACT-Ntx.

Table 10. CFA factor loadings and squared multiple correlations for CIPNAT scales

Table 11. Goodness of fit indices

Table 12. Cronbach's alpha for CIPNAT and FACT-Ntx 


\section{List of Figures}

Figure 1. Conceptual Framework based on Theory of Unpleasant Symptoms 9

Figure 2. Original Peripheral Neuropathy Scale 13

Figure 3. GOG Performance Status Scale 15 
Psychometric Evaluation of the Chemotherapy Induced Peripheral Neuropathy Assessment Tool

Cindy Tofthagen

\begin{abstract}
Chemotherapy induced peripheral neuropathy (CIPN) is a common side effect of several chemotherapy drugs used for the treatment of many common malignancies. CIPN is both under-assessed and underreported and few self-report tools exist that measure CIPN. Existing instruments do not evaluate all of the multi-dimensional characteristics of neuropathic symptoms; intensity, distress, timing, and characteristics. The purpose of this descriptive, cross-sectional study was to develop and psychometrically evaluate a new self - report tool for CIPN, the Chemotherapy Induced Peripheral Neuropathy Assessment Tool (CIPNAT). Interviews with 15 patients with known CIPN guided development of the CIPNAT.

The CIPNAT is a 69 item self-report tool which measures CIPN, including characteristics, intensity, distress, frequency, and interference with usual activities. Content validity was evaluated by a panel of experts and revisions were made to the CIPNAT based on those results. The CIPNAT was administered to 167 patients on chemotherapy at H. Lee Moffitt Cancer Center and a two physician medical oncology practice in Tampa, Florida. The Functional Assessment of Cancer Therapy-Neurotoxicity scale (FACT/GOG-Ntx), another self-report tool for CIPN was also administered.
\end{abstract}


Correlations between the CIPNAT and the FACT/GOG-Ntx were evaluated. Differences between a group of 40 patients getting non-neurotoxic chemotherapy and a group of 127 patients getting neurotoxic chemotherapy were also examined. Test-retest reliability was evaluated by administering the CIPNAT a second time to a subgroup of 30 patients and correlating the results. Correlation with a measure of the same concept indicated that scores between the CIPNAT and the FACT-Ntx were strong $(\mathrm{r}=.73, \mathrm{p}=.000$, $\mathrm{n}=127)$.Differences between the two contrasting groups were significant $(\mathrm{p}=.000)$, supporting validity. High test-retest correlations $(r=.921, p=.000)$ demonstrated reliability. Cronbach's alpha for the total CIPNAT (alpha=.945), the symptom experience scale (alpha $=.927)$ and the interference scale (alpha=.897) demonstrated high internal consistency reliability. Confirmatory factor analysis of neuropathic symptoms indicated the presence of two underlying factors, sensory symptoms and motor symptoms. Confirmatory factor analysis of the interference scale also indicated two underlying factors, activities requiring manual dexterity and general activities. These results provide strong evidence of the validity and reliability of the CIPNAT. 


\section{Chapter One}

Introduction

Chemotherapy induced peripheral neuropathy (CIPN) is a group of

neuromuscular symptoms that result from nerve damage caused by drug therapies used in the treatment of cancer (Visovsky, 2003). Peripheral neuropathies are one of the most frequently occurring and distressing side effects of chemotherapy (Visovsky, 2003; Wickham, 2007). More than $30 \%$ of patients who receive paclitaxel, docetaxel, bortezomib, thalidomide, or oxaliplatin will develop chemotherapy induced peripheral neuropathy (CIPN). Several other drugs commonly used in the fight against cancer cause CIPN in 10-29\% of patients (University of Florida Shands Cancer Center, 2007). The development of new anticancer therapies has led to increased survival (American Cancer Society, 2008); but several of these new therapies are toxic to the nervous system, causing a variety of unpleasant symptoms that are uncomfortable and difficult to manage (Bakitas, 2007; Wilkes, 2007). Severe peripheral neuropathies result in dose reductions, a change in chemotherapy regimen, or early cessation of chemotherapy, all of which compromise the success of cancer treatment (Mantyh, 2006). In addition, neuropathies can interfere with key aspects of quality of life including physical, social, and role functioning and emotional well being (Bakitas, 2007; Ostchega, Donohue, \& Fox, 1988). 


\section{Underlying Mechanisms}

The mechanisms that cause CIPN are not well understood. Large diameter neurons are surrounded by an insulating layer, or myelin sheath, and are called either Aalpha fibers or A-beta fibers (Kasper et al., 2005). The myelin sheath helps facilitate transmission of impulses along the nerve. Smaller diameter neurons are called C fibers or A-delta fibers and lack a myelin sheath (Kasper et al., 2005). Many chemotherapy agents are believed to cause destruction or dysfunction of the myelin sheath. This may cause abnormal burning, prickling, or tingling sensations called paresthesias. Loss of vibratory sense, two-point discrimination, and proprioception may also result from damage to the

myelin sheath (Mantyh, 2006). Destruction or dysfunction of C and A-delta fibers results in muscle pain, burning pain in the extremities, and increased sensitivity to cold. Damage to the nervous system is believed to cause sensitization and hyperexcitability of peripheral nociceptors and hyperexcitability of the dorsal horn (Baron, 2006). Severe and irreversible sensory neuropathies can be the result of axonal degeneration and injury to the dorsal root ganglion and are associated with neurotoxic chemotherapies such as cisplatin, paclitaxel, and oxaliplatin (Ocean \& Vahdat, 2004).

\section{Risk Factors and Symptoms}

The biggest risk factor for the development of CIPN is treatment with neurotoxic anti-cancer drugs (Ostchega et al., 1988; Visovsky, C, 2003; Wickham, 2007; Wilkes, 2007). Risk of developing CIPN rises as the cumulative dose of chemotherapy escalates (Lipton et al., 1989; Pietrangeli, Leandri, Terzoli, Jandolo, \& Garufi, 2006; Quasthoff \& Hartung, 2002). People who receive two or more neurotoxic chemotherapy drugs are at 
higher risk as are those with preexisting neuropathy (Badros et al., 2007; Chaudhry, Chaudhry, Crawford, Simmons-O'Brien, \& Griffin, 2003; Gordon, Stringer, Matthews, Willis, \& Nemunaitis, 1997; Huang, Brady, Cella, \& Fleming, 2007).

Symptoms of CIPN may include numbness and/or tingling beginning in the tips of the fingers or toes ( Ocean \& Vahdat, 2004; Wilkes, 2007). Burning, shooting, or electric like pain, prickling sensation, loss of touch and temperature discrimination, hyporeflexia, and muscle weakness also are reported. Symptoms become more pronounced with escalating doses of the drug, frequently resulting in discontinuation of the drug before maximum benefit is achieved (Dunlap \& Paice, 2006). Although symptoms usually subside when the neurotoxic drug is stopped, permanent damage to the nervous system can occur (Ocean \& Vahdat, 2004). CIPN is associated with decreased ability to perform activities of daily living, psychological distress and reduced satisfaction with life (Ostchega, Donohue, \& Fox, 1988).

Neuropathies can be categorized as either sensory or motor symptoms (Quasthoff \& Hartung, 2002). Motor symptoms affect skeletal muscles. Motor symptoms include arthralgias, myalgias, muscle weakness, and loss of balance. Sensory nerves affect sensation and provide information about touch, pain, temperature, vibration and position (Sweeney, 2002). Sensory symptoms may include numbness, tingling, neuropathic pain, and sensitivity to cold or heat.

Neuropathic symptoms can also be classified as painful or non-painful (Empl et al., 2001) . Painful symptoms involve exaggerated or unusual sensations while nonpainful symptoms involve loss of sensation (Kasper et al., 2005). Other authors have used 
the terms positive and negative (Kasper et al., 2005), ataxic and painful, and painful and painless (Uceyler, Rogausch, Toyka, \& Sommer, 2007) to similarly classify neuropathic symptoms.

\section{Definition of Terms}

Chemotherapy induced peripheral neuropathy. Chemotherapy induced peripheral neuropathy refers to a group of neuromuscular symptoms that result from nerve damage caused by drug therapies used in the treatment of cancer (Visovsky, 2003). Manifestations of chemotherapy induced peripheral neuropathy include painful neuropathic symptoms, non-painful neuropathic symptoms, and interference with usual activities.

Painful neuropathy. Painful neuropathy refers to a group of neurological symptoms characterized by pain, discomfort, or exaggerated sensation. Painful neuropathy also can be referred to as neuropathic pain (Backonja, 2003). The International Association for the Study of Pain (IASP) defines neuropathic pain as "pain initiated or caused by a primary lesion or dysfunction of the nervous system"(Merskey \& Bogduk, 1994, p.209-214). Painful neuropathy can be described using a variety of terms including sharp, tingling, aching, burning, shooting, or electric-like but not all patients with CIPN identify uncomfortable neurologic symptoms as painful (Smith, Whedon, \& Bookbinder, 2002).

Non-painful neuropathy. Non-painful neuropathy refers to a group of neurological symptoms characterized by loss of sensation or loss of function. Numbness, muscle weakness, loss of deep tendon reflexes, loss of balance, and loss of fine motor skills are 
all examples of non-painful neuropathy. Non-painful neuropathy is thought to have a greater effect on physical function than painful neuropathy (Empl et al., 2001).

Symptom interference. Symptom interference with usual activity refers to the extent to which symptoms interfere with activities that are necessary to meet basic needs and maintain health and well-being (Leidy, 1994). CIPN symptoms are believed to have a negative impact on patients' ability to perform their usual activities by negatively affecting physical performance (Almadrones, McGuire, Walczak, Florio, \& Tian, 2004; Bakitas, 2007; Smith et al., 2002; Visovsky, 2003). Physical performance is an important aspect of quality of life for individuals with cancer (Ferreira et al., 2008).

\section{Measurement}

Lack of a "gold standard" assessment technique, inconsistent use of terminology, lack of psychometric evidence to support the use of self-report instruments in cancer populations, and lack of attention to multidimensional characteristics are some of the limitations associated with CIPN (Dunlap \& Paice, 2006). Comprehensive assessment of CIPN is a critical step toward the development of interventions to promote safety, alleviate symptoms, and maximize quality of life for persons with cancer. Two general approaches to assessment are discussed in the existing literature. One approach is clinician evaluation, and the second approach is patient self-report.

Clinical evaluation. Clinician evaluation is accomplished through complete neurologic examination, nerve conduction studies, and the use of grading scales. Assessment via complete neurological exam and nerve conduction studies can yield useful information; however results of such testing often do not correlate with patient 
report and do not adequately portray the symptom experience (Calhoun et al., 2003). Grading scales often are used in research and practice. The World Health Organization, Eastern Cooperative Oncology Group and National Cancer Institute all have developed grading scales for CIPN (Oncology Nursing Society, 2005). Scores for these scales are calculated by clinicians based on patient report and physical exam. Grading scales, while useful, have many limitations including their uni-dimensional nature and lack of sensitivity and specificity (Wickham, 2007).

Performance measures designed to help clinicians assess neuropathic symptoms have been developed, including the Total Neuropathy Score, the Neuropathy Symptom Profile, and the Neurological Disability Score. Results of these scales can be subjective, dependent upon the expertise of the examiner and only address prevalence of symptoms. They do not provide information on severity, distress, timing, or impact on daily activities (Postma et al., 2005).

Self-report. Self-report is a critical element of evaluation of CIPN. Few reliable and valid instruments for CIPN are currently available. Existing instruments, though well designed and psychometrically sound, do not capture the multidimensional experience of CIPN. Some instruments assess neuropathic pain but are not specific to chemotherapy induced peripheral neuropathy. Others do not include evaluation of non-painful symptoms or motor symptoms. Most of these instruments measure the presence of symptoms, attempt to evaluate physical dysfunction related to neuropathies, and some also assess quality of life, but none of the instruments assess intensity, timing, or distress caused by CIPN. Furthermore, their assessment of symptom interference is not 
comprehensive enough to give clinicians and researchers enough information to guide clinical decision making. A more detailed evaluation of individual symptoms and symptom interference would provide more information to help clinicians suggest interventions designed to maximize patient safety and physical performance. It would also allow researchers to evaluate the efficacy of such interventions. The challenge in developing a more comprehensive self report tool for CIPN is to make it brief and easy to administer (Dunlap \& Paice, 2006).

A detailed discussion of the psychometric properties of the instruments available for CIPN follows in the review of literature.

\section{Statement of the Problem and Purpose}

Researchers interested in studying chemotherapy induced peripheral neuropathy need more scientifically validated measurement tools. Several tools have been developed but many measurement issues still exist in the study of CIPN including lack of a "gold standard" assessment technique, lack of psychometric evidence to support the use of selfreport instruments in cancer populations, and lack of attention to the multidimensional characteristics of CIPN (Dunlap \& Paice, 2006).

The purpose of the study is to evaluate the psychometric properties of an instrument designed to measure CIPN, the Chemotherapy Induced Peripheral Neuropathy Assessment Tool (CIPNAT) (Appendix B). Assessment of symptoms included presence of neuropathic symptoms, intensity, distress, timing of symptoms, and impact of symptoms on daily activities. Psychometric evaluation included assessment of content validity, construct validity, test-retest reliability, and internal consistency reliability. 
Development and testing of this instrument was conducted in three phases. Phase I was a mixed methods analysis of interviews with 15 patients with CIPN. The methods and quantitative results are discussed in Chapter Three. Phase II involved having a panel of five experts review the content of the CIPNAT and provide feedback on the relevance of each item. Revisions to the CIPNAT were made based on feedback from the panel of experts. Phase II methods and results are discussed in Chapter Four. Phase III involved administering the CIPNAT to a group of patients in order to evaluate its reliability and validity in cancer patients receiving chemotherapy. Phase III methods and results are discussed in Chapter Five.

\section{Conceptual Framework}

The conceptual framework for this study is based upon the Theory of Unpleasant Symptoms (Lenz, Pugh, Milligan, Gift, \& Suppe, 1997; Lenz, Suppe, Gift, Pugh, \& Milligan, 1995). Physical, psychological, and social factors all influence the symptom experience. The physical factors that influence neuropathic symptoms include chemotherapy drug used, the treatment schedule, and the cumulative dose (Lenz, et al., 1997) (Figure 1). The authors conceptualized unpleasant symptoms like CIPN to be multidimensional in nature (Lenz, et al., 1997). Character, timing, intensity, and distress are important aspects of the symptom experience, and evaluation of these dimensions will lead to a better understanding of meaningful aspects of CIPN.

Characteristics. Characteristics of CIPN vary from person to person and are influenced in part by which chemotherapy drug patients receive. Patients may experience one or more neuropathic symptoms at the same time and may develop more neuropathic 
symptoms over time. The presence of more neuropathic symptoms indicates more severe CIPN.

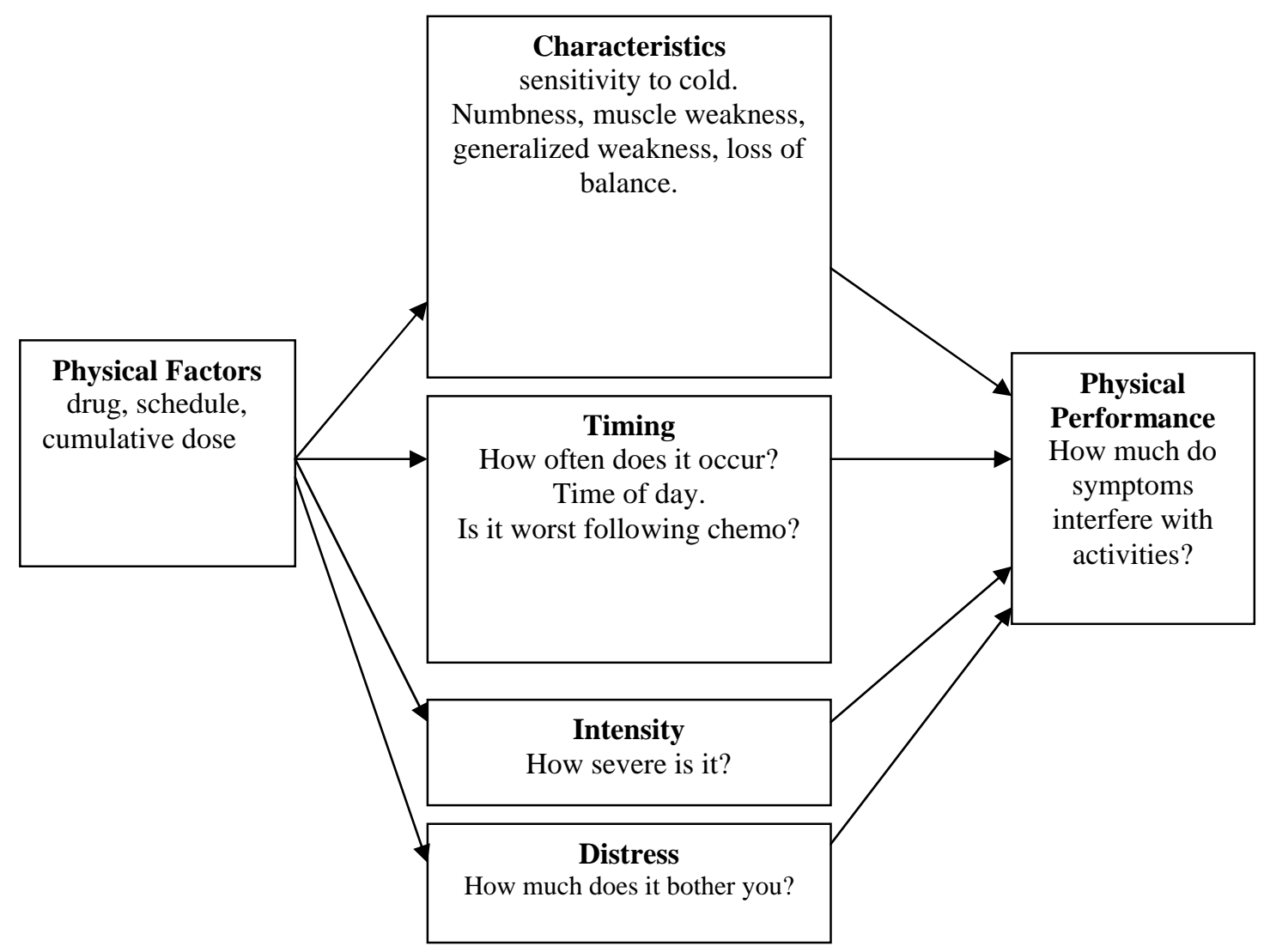

Figure 1. Conceptual framework based on Theory of Unpleasant Symptoms.

Timing. Timing of neuropathic symptoms includes the time of day symptoms occur or are most severe, whether symptoms are intermittent or continuous, and whether they are most severe following chemotherapy. Certain neuropathic symptoms are more likely to be continuous. Numbness and generalized weakness are conceptualized as gradually progressing over time but once they occur, these symptoms are likely to be continuous. Other symptoms like pain, cold sensitivity, and arthralgias/myalgias may be 
more likely to occur in the days following chemotherapy. Continuous symptoms may signify more severe neuropathy than intermittent symptoms.

Intensity. Intensity refers to how severe patients perceive their symptoms and may be useful in evaluating changes in symptoms over time. It is possible for two people to report the same level of intensity but have very different levels of distress. More severe individual symptoms contribute to higher levels of CIPN but alone do not provide enough information to assess overall severity of neuropathic symptoms.

Distress. Distress is an emotional component of the symptom experience. It signifies how emotionally troubled someone is by the symptoms they are experiencing. Many factors can influence distress level such as other life stressors, competing demands, and limited physical performance. For example, a man who plays an instrument for a living who finds he is gradually losing the manual dexterity needed to play his instrument will probably experience more distress over numbness in the hands than he will from numbness in the feet. Another individual who enjoys running but is now experiencing numbness in the feet may be more bothered by that symptom.

Physical performance. Character, intensity, distress and timing should be included in assessment of CIPN because these elements influence physical performance. Neuropathic influence on physical performance can be assessed by evaluating to what extent the ability to participate in usual activities has been affected by neuropathic symptoms. It also is important to determine which activities may be adversely affected because when physical performance is impaired, it may threaten patients' independence and negatively influence self-concept. 


\section{Significance to Nursing}

Nurses are instrumental in providing symptom management to oncology patients (Smith et al., 2002). Well designed instruments should help nurses more thoroughly assess symptoms. Adequate assessment is critical in planning nursing interventions to best meet the needs of the patient. Psychometrically sound measurement tools that better capture the full experience of patients are needed. Currently, there are no instruments for CIPN that evaluate character, intensity, distress, timing, and impact on physical functioning. Development of such an instrument would allow clinicians and researchers to thoroughly evaluate the impact of peripheral neuropathies on physical and emotional outcomes. More detailed evaluation of CIPN will help nurses and other health care professionals design and implement interventions to help prevent symptoms, recognize symptoms earlier, and treat symptoms more effectively. 


\section{Chapter Two}

Review of the Literature

Subjective measures may provide more useful information regarding symptoms than physical exam (Calhoun, et al., 2003). A few self-report tools have been developed to help quantify symptoms of CIPN. They are useful for quantifying the presence of neuropathic symptoms but do not adequately assess emotional distress caused by neuropathic symptoms, timing of symptoms, or effects of symptoms on physical function. Studies evaluating the psychometric properties of existing instruments are discussed in the remainder of this chapter.

\section{Peripheral Neuropathy Scale}

Ostchega, Donohue, and Fox (1988) were the first nurse researchers to design a self report tool to assess CIPN and describe the affects of CIPN on daily functioning. Using a modified version of the European Organization for Treatment of Cancer Quality of Life Questionnaire (EORTC QOLQ) they devised an instrument intended to reflect common complaints associated with CIPN (Figure 2). The Peripheral Neuropathy Scale (PNS) consisted of five items evaluating the presence of numbness, tingling, pain, and stiffness or tightness in the hands and feet and three items addressing physical function. A four point rating system included possible responses of not at all $=1$, a little $=2$, quite a bit $=$ 3 , and very much $=4$. Content validity for the PNS was evaluated by a panel of experts 
made up of two patients with CIPN and two physicians with knowledge of CIPN symptoms. No other validity or reliability testing was reported (Ostchega et al., 1988).

During the last three days:

1. Did you have any difficulty buttoning buttons?

2. Did you have any stiffness or tightness in your hands or feet?

3. Did you feel clumsy?

4. Did you feel pain in your hands?

5. Did you feel pain in your feet?

6. When holding an object in your hand, were you able to feel its shape?

7. Did you have tingling in your hands or feet?

8. Did you have numbness in your hands or feet?

Figure 2. Original Peripheral Neuropathy Scale.

The authors of this instrument conducted a descriptive retrospective study involving 30 people with CIPN. Ten were women who had been treated with cisplatin for ovarian cancer and 20 were men who had been treated with cisplatin for testicular cancer. Five of the women had completed cisplatin therapy less than one year before the interviews. The rest of the participants had completed cisplatin therapy from 1-4 years prior to the interview. The goals of the study were to: 1) explore the effects of CIPN on quality of life 2) determine if symptoms decrease over time, and 3) determine factors that predict variability in symptoms. Data were collected by phone interview or personal 
interview using the EORTC QOLQ, the Satisfaction with Life Scale (SWLS) and the PNS. The study demonstrated that more severe CIPN is positively correlated with fatigue and malaise $(\mathrm{r}=.69, \mathrm{p}<.001)$, psychological distress $(\mathrm{r}=.36, \mathrm{p}<.05)$ and negatively correlated with subjective sense of well-being $(-.52, \mathrm{p}<.001)$. Participants with severe symptoms were less able to perform ADL's $(\mathrm{p}=.00)$ and usual work $(\mathrm{p}=.05)$ than those with mild symptoms. In addition, time since treatment was associated with less severe symptoms (r=-.48, p<.05) (Ostchega et al., 1988).

The PNS was the first scale designed to measure CIPN but it had many problems that limited its usefulness in further research. Items were worded in the past tense, possibly causing confusion to participants who are actively experiencing symptoms. Reliability and validity were not thoroughly evaluated by the authors. In addition, the PNS evaluates character of neuropathic symptoms, but motor symptoms like muscle weakness and muscle pain were not included, presumably because these are not common neurologic toxicities of cisplatin. The three items evaluating physical function; difficulty buttoning, difficulty feeling the shape of an object in the hand, and clumsiness failed to capture the wide range of physical limitations that CIPN can produce.

Sixteen years later, Almadrones and colleagues (2004) revised the PNS and evaluated psychometric properties in 88 women receiving chemotherapy for ovarian cancer. This time the PNS was combined with items from the Gynecologic Oncology Group Performance Status Scale (Figure 3) to more thoroughly evaluate aspects of physical function related to CIPN. The same four point system for rating presence of symptoms used by Ostchega, Donohue and Fox (1988) was retained. The original PNS 
was expanded to 11 items by separating hand symptoms from foot symptoms and items worded using past tense were reworded to reflect present tense. The resulting PNS contained 11 items and scores ranged from 11 to 44 with higher scores corresponding with more severe neuropathic symptoms. Scores on the GOG Performance Status Scale ranged from 8-40 with higher scores indicating more physical limitations (Almadrones, McGuire, Walczak, Florio, \& Tian, 2004).

\section{Physical Function}

1. Do you need help with eating, dressing, washing, or using the toilet?

2. Do you have to stay indoors most or all of the day?

3. Are you in bed or a chair most of the day?

4. Do you have trouble either walking a short distance or climbing one flight of stairs?

5. Do you have trouble bending, lifting, or stooping?

6. Do you have trouble either taking a walk or climbing a few flights of stairs?

\section{Role Function}

7. Does your condition keep you from working at a job or doing household jobs?

8. Are you limited in any way doing your work or household jobs?

Figure 3. GOG Performance Status Scale.

Participants in this study were part of a phase III clinical trial comparing the efficacy and tolerability of six cycles of cisplatin and cyclophosphamide to six cycles of 
cisplatin and paclitaxel. Questionnaires were administered to patients before chemotherapy was initiated (T1) and again at the end of six cycles of chemotherapy (T2). Sixty-seven patients completed the questionnaires at the end of the study. Internal consistency reliability was evaluated using Cronbach's alpha. Cronbach's alpha coefficients before chemotherapy and after six cycles of chemotherapy demonstrated internal consistency for the peripheral neuropathy scale (alpha $=.91-.92)$, physical function (alpha $=.89-.91)$, and role function scales $($ alpha $=.92-.96)$. Two items in the peripheral neuropathy scale, difficulty buttoning $(r=.66$ at $\mathrm{T} 1$ and $\mathrm{r}=.36$ at $\mathrm{T} 2)$ and difficulty feeling objects in the hand $(\mathrm{r}=.26$ at $\mathrm{T} 1$ and $\mathrm{r}=.31$ at $\mathrm{T} 2)$ were not highly correlated with total scores. Construct validity was assessed at T2 by correlating scores on the GOG toxicity criteria for peripheral neuropathy to scores on the PNS using Spearman's rank correlation test of association. Results support the PNS as a valid measure of peripheral neuropathy. Exploratory factor analysis was used to evaluate construct validity. Results suggest that the peripheral neuropathy scale is comprised of hand neuropathy and foot neuropathy subscales (Almadrones et al., 2004).

The revised PNS is better designed and has undergone one study evaluating its psychometric properties but still does not thoroughly evaluate neuropathic symptoms. No items evaluating motor symptoms were added. The PNP/GOG Performance Status Scale still does not assess physical function specifically enough to provide meaningful information. It includes general measures of physical function that are likely to be affected by cancer symptoms or treatment related fatigue. It does not assess the areas of daily functioning that are most likely to be affected by CIPN like driving, picking up 
objects, or writing. In addition, it does not assess symptom severity, symptom distress, or timing of symptoms.

\section{FACT/GOG Neurotoxicity Subscale}

The FACT/GOG neurotoxicity scale (FACT/GOG-Ntx) was developed in 1998 through a collaboration between the Gynecologic Oncology Group (GOG) and the authors of the Functional Assessment of Chronic Illness Therapy (FACT) (Calhoun et al., 2003) (Appendix A). The FACT/GOG-Ntx evaluates health related quality of life in cancer patients with CIPN. A four point rating system with possible responses include 0 $=$ not at all, $1=$ a little bit, $2=$ somewhat, $3=$ quite a bit, and $4=$ very much is used (Calhoun, et al., 2003).

Initial psychometric evaluation of the FACT/GOG-Ntx involved data from two studies of women with ovarian cancer. One group of 60 women receiving paclitaxel and carboplatin completed the FACT/GOG-Ntx and also received a complete neurologic exam. Neurologic examination was completed before the first cycle of chemotherapy and this group served as a reference group. All participants in the second group of 43 patients had grade two or higher peripheral neuropathy, numbness in hands/feet, hearing loss or blurred vision. They also completed the FACT/GOG-Ntx. Both groups were reassessed at 3 month intervals until 12 months following completion of chemotherapy. Scores between the two groups were then compared in order to evaluate discriminate validity. Responsiveness, or sensitivity to change, was evaluated by comparing changes over time between the two groups. Construct validity was evaluated by examining correlations between FACT/GOG-Ntx scores and neurologic exam data (Calhoun, et al., 2003). 
Cronbach's alpha coefficients for the neurotoxicity subscale exceeded the predetermined level of .70 in 11 out of 12 assessments demonstrating internal consistency reliability. Mean scores between the two groups on the neurotoxicity subscale differed significantly at baseline, 3 month follow-up and 6 month follow-up, demonstrating responsiveness over time. Correlations between FACT/GOG-Ntx scores and some elements of neurologic exam data were significant, while others were not. Other studies have also shown that patient report does not correlate well with neurologic exam (Calhoun et al., 2003).

The FACT/GOG-Ntx was modified for use in patients receiving oxaliplatin by Kopec and colleagues (2006). Two additional items pertaining to sensitivity to cold were added and the resulting scale was administered to 395 patients with colon cancer. Participants received either a non-neurotoxic chemotherapy regimen with leucovorin and 5-fluorouracil $(\mathrm{n}=206)$ or a neurotoxic regimen including leucovorin, 5-fluorouracil and oxaliplatin $(\mathrm{n}=189)$. Neurotoxicities were also assessed using The National Cancer Institute's (NCI) common toxicity criteria for peripheral neuropathy. This is a clinician rated assessment with scores as follows: $0=$ normal, $1=$ loss of tendon reflexes or paresthesia (including tingling) interfering with function but not activities of daily living, $3=$ sensory loss or paresthesia interfering with activities of daily living or $4=$ permanent sensory loss that interferes with function. Questionnaires were completed at baseline, 3 months after initiation of chemotherapy, and at 6,12 , and 18 months following initiation of chemotherapy (Kopec et al., 2006). 
Cronbach's alpha for the new scale at the 6 month measurement was 0.85 . Factor analysis yielded a single factor solution, indicating that the scale is uni-dimensional. Correlations between items and between each item and the total score were calculated. Relationships between each item and the NCI common toxicity criteria were calculated. Trouble hearing, ringing or buzzing in the ears and difficulty breathing when exposed to cold, and feeling weak all over had correlations less than 0.2 with the NCI common toxicity criteria. Trouble hearing, ringing or buzzing in the ears and difficulty breathing when exposed to cold had item-total correlations less than 0.4. Difficulty breathing when exposed to cold was removed from the scale based on those results. Sensitivity to change was assessed by the effect size (mean scores/standard deviation) for changes from baseline to the 3 month assessment. An effect size greater than 0.8 signifies a large effect. The effect size for changes in scores was .83 for the original scale and 1.10 for the final scale (Kopec et al., 2006).

A third study evaluating the psychometric properties of the FACT/GOG-Ntx was conducted with 116 chemotherapy-naive women being treated with cisplatin, doxorubicin, and paclitaxel for endometrial cancer (Huang et al., 2007). Participants completed the FACT/GOG-Ntx prior to administration of each chemotherapy cycle for up to seven cycles. The National Cancer Institute's (NCI) Common Toxicity Criteria for peripheral neuropathy was also calculated by a clinician before each chemotherapy cycle. Principal component analysis was used to determine the number of latent constructs. The authors concluded that the 11 item neurotoxicity subscale assesses four latent constructs: 
sensory neuropathy, motor neuropathy, hearing neuropathy, and neuropathy related dysfunction (Huang et al., 2007).

Internal consistency reliability was assessed using Cronbach's alpha. Values ranged from 0.80 to 0.85 for the total scale and between 0.7 and .91 for an abbreviated four item version of the scale. Construct validity was assessed by examining item-total correlations. All correlations were weak to moderate $(\mathrm{r}=0.3-0.4)$ at baseline. Correlations between the sensory neuropathy items and the total score got progressively stronger, reaching $0.6-0.8$ by the end of the study. Other item-total correlations either remained the same or were slightly increased by the end of the study. Significant differences between a second group of patients $(n=129)$ receiving a less neurotoxic chemotherapy regimen (cisplatin and doxorubicin only) were also used as evidence of construct validity.

Differences between mean scores from baseline (3.67) to before the seventh cycle (8.13) were significant, demonstrating responsiveness of the subscale to changes over time (Huang et al., 2007).

Four items accounted for $80 \%$ of the variance and $63 \%$ of changes in the subscale over time. These items made up the sensory subscale and were as follows: 1) numbness or tingling in the hands 2) numbness or tingling in the feet 3) discomfort in the hands 4) discomfort in the feet. The authors concluded that when patient burden is a concern, the abbreviated four item version may be used. The abbreviated four item scale allows for quick assessment but may not be responsive to changes over time or provide enough information to accurately capture the entire symptom experience (Huang et al., 2007). 
Cella and colleagues (2003) added five items for assessment of taxane related side effects to the FACT/GOG-Ntx subscale. They evaluated the psychometric properties of the new scale on 240 patients receiving paclitaxel and carboplatin for treatment of nonsmall cell lung cancer (Cella, Peterman, Hudgens, Webster, \& Socinski, 2003). The resulting taxane specific scale is referred to as the FACT-taxane. Additional items pertained to feeling bloated, having edema of the upper or lower extremities, having pain in the fingertips or feeling bothered by the appearance of hands or nails.

Questionnaires were administered at baseline and at 6, 12, and 26 weeks and included the FACT-taxane and the Functional Assessment of Cancer Therapy-General (FACT-G), a general measure of health related quality of life for people with cancer. Internal consistency reliability of the FACT/GOG-Ntx was demonstrated again, with Cronbach's alpha coefficients ranging from .82 to .86 for the FACT/GOG-Ntx and .84.88 for the taxane specific items. Responsiveness to change over time was supported by the findings of significant worsening of neurotoxic symptoms from baseline to the six week assessment $(\mathrm{p}=0.000)$ as well as from the six week to the twelve week assessment $(\mathrm{p}=0.000)($ Cella et al., 2003).

The FACT/GOG neurotoxicity subscale is without a doubt the most widely used and thoroughly evaluated instrument available for CIPN. Unfortunately, multiple changes to the scale have been required based on the population being studied and which neurotoxic agents were administered. There is a need for an instrument that can be used across populations receiving different neurotoxic chemotherapies without a need to change the contents of the scale. Some of the items evaluating physical function 
including trouble buttoning buttons and feeling the shapes of objects in the hands have had low item-total correlations in prior studies. The hearing neuropathy items have also shown low item-total correlations in previous studies. The neuropathy related dysfunction subscale fails to evaluate common aspects of physical performance affected by CIPN and the instrument does not evaluate timing of symptoms, or symptom distress.

Quality of Life Questionnaire-Chemotherapy Induced Peripheral Neuropathy 20

The Quality of Life Questionnaire-Chemotherapy Induced Peripheral Neuropathy 20 (QLQ-CIPN20) is another neuropathy scale designed to supplement one of the EORTC Quality of Life questionnaires (Postma et al., 2005). It was developed based on results of a literature search, a panel of experts, and interviews with 68 patients with a variety of malignancies receiving neurotoxic chemotherapy. The most prevalent symptoms reported by patients included numbness and tingling, problems with standing or walking, difficulty opening a bottle or jar, difficulty getting out of a chair, and leg weakness. The resulting QLQ-CIPN20 contains three scales evaluating sensory, motor, and autonomic symptoms and functioning. It was pre-tested on 44 participants in Europe and demonstrated adequate internal consistency reliability for the sensory scale (Cronbach's alpha 0.82), the motor scale (Cronbach's alpha 0.73), and the autonomic scale (Cronbach's alpha 0.76). The participants had all received taxanes, platinum-based drugs, or vinca-alkaloids (Postma et al., 2005). More extensive psychometric evaluation is in progress but no other studies of the QLQ-CIPN20 were found in a recent search of PubMed. This scale needs further evaluation of psychometric properties before it can be considered a valid tool for measuring CIPN. 


\section{Summary}

In summary, a review of the literature resulted in identification of three self-report instruments for chemotherapy induced peripheral neuropathy. The instrument that was identified most frequently was the FACT/GOG-Ntx. It has been the subject of at least four studies which support its use as a reliable and valid instrument. Reliability and validity of the PNS and QLQ-CIPN20 also have been evaluated but studies to date have involved small samples. None of the instruments thoroughly evaluate key aspects of CIPN, particularly timing of symptoms, severity of symptoms or interference with physical functioning.

These studies support the inclusion of numbness, tingling, pain or discomfort, arthralgias/myalgias, loss of balance, muscle weakness, generalized weakness, cold sensitivity, and interference with usual activities in assessment of CIPN and confirm the need for development of new, more comprehensive self- report tools for CIPN. Such an instrument should be applicable to patients receiving any type of neurotoxic chemotherapy, should specifically assess timing of symptoms, address the individual level of distress that each symptom causes for the patient, and should thoroughly evaluate neuropathic impairment with physical function. 


\section{Chapter Three}

Phase I Method, Results and Discussion

The first phase of instrument development was to learn, from patients who were currently experiencing CIPN, how they describe symptoms, how symptoms affect their daily lives, and what they found most bothersome about the experience of CIPN. A qualitative study of patients with chemotherapy induced peripheral neuropathy was conducted between August of 2007 and February of 2008. The methods and outcomes of this study are presented in this chapter.

\section{Method}

Sample and Setting. The researcher conducted semi-structured interviews with 15 participants who had neuropathic symptoms and who had been treated with taxanes, platinum-based drugs, vinca alkaloids, or thalidomide within the previous three years. Approval for the study was obtained from the Institutional Review Board (IRB) of the University of South Florida. Informed consent was obtained. Participants were recruited from an outpatient medical oncology clinic in Central Florida, where the author of this manuscript works as a nurse practitioner and spent 12 years as a chemotherapy nurse. Many of the participants were previously known to the investigator.

Procedures. Semi-structured interviews were conducted with the participants while they were at the clinic, were recorded, and professionally transcribed. Patients were asked the following questions: 
1. Tell me about any of the following symptoms that you have experienced since starting chemotherapy: numbness, tingling, sharp, stabbing or throbbing pain, a "pins and needles" sensation or feeling like part of your body is "asleep".

2. What other words would you use to describe these symptoms?

3. How have these symptoms affected your daily life?

4. What things have these symptoms interfered with your ability to do?

5. What do you find most troubling about these symptoms?

6. Is there anything else you would like to share with me about these symptoms?

Relevant comments made by participants during the course of the interview were recorded. Demographic information was obtained from the medical record including age, gender, cancer type, name and cumulative dose of neurotoxic chemotherapy drug used and whether chemotherapy doses were altered or chemotherapy was interrupted as a result of peripheral neuropathy.

Transcripts were reviewed numerous times for relevant content. Each transcript was examined for neuropathic symptoms and descriptors. Each descriptor was entered as a variable into a database and coded as a one if the patient reported it or a zero if they did not report it. Terms that described similar concepts were combined. For instance, patients used a variety of terminology to describe loss of balance including "clumsiness", "loss of balance", "dizzy in the head" and "not being able to walk". These were categorized together as "loss of balance". Data were analyzed using the Statistical Software Package for the Social Sciences (SPSS) version 16.0 and confirmed using Atlas TI. Descriptive statistics including frequencies and percentages were analyzed. Items for instrument 
development were selected from the quantitative analysis reported below. The preliminary version of the CIPNAT was developed based upon the results of this study and is supported by existing literature on neuropathic symptoms.

Results

Demographics. The sample was almost equally divided between men and women with 8 males and 7 females ranging in age from 42 to 84 . Participants were primarily Caucasian and had lung cancer, breast cancer, colon cancer, multiple myeloma or cholangiocarcinoma. The majority were treated with taxanes or oxaliplatin (Table 1). Over fifty-three percent had not yet had a dose reduction or discontinuation of treatment while $46.6 \%$ had dose reductions or discontinuation of the neurotoxic agent at the time of the interview.

Table 1. Frequencies and percentages for race, gender, cancer type and neurotoxic agent.

\begin{tabular}{llc}
\hline Variable & $N$ & Percentage \\
\hline Race & & \\
$\quad$ White/Caucasian & 14 & 93 \\
$\quad$ African-American & 1 & 7 \\
Gender & & \\
Male & 8 & 53 \\
$\quad$ Female & 7 & 47 \\
Cancer Type & & \\
Lung & 4 & 27 \\
Breast & 4 & 27 \\
Colorectal & 4 & 27 \\
Multiple Myeloma & 2 & 13 \\
Cholangeocarcinoma & 1 & 7 \\
Neurotoxic agent & & \\
paclitaxel & 4 & 27 \\
docetaxel & 3 & 20 \\
$\quad$ vinorelbine & 1 & 7 \\
$\quad$ oxaliplatin & 5 & 33 \\
thalidomide & 2 & 13 \\
\hline
\end{tabular}


Borderline case. One borderline case described symptoms that were dissimilar to the other patients. Her symptoms were also inconsistent with the literature on chemotherapy induced neuropathy. She had mild, temporary neurological symptoms following her first treatment with oxaliplatin. These symptoms subsided within days after the first treatment. Her second treatment resulted in immediate acute onset of shortness of breath and anxiety probably attributable to oxaliplatin induced laryngopharyngeal spasm.

Non-painful symptoms. Patients reported a variety of non-painful symptoms including numbness, short term memory loss, trouble concentrating, loss of balance, muscle weakness, clumsiness, loss of depth perception, lack of coordination, dizziness and generalized weakness (Table 2). Of these symptoms, numbness, muscle weakness, loss of balance, and generalized weakness were reported by at least 33\% of participants. All of the patients except the borderline case reported numbness of the fingers and/or toes. Patients frequently described simultaneous pain or discomfort and numbness.

Table 2. Frequencies and percentages for non-painful symptoms.

\begin{tabular}{llc}
\cline { 3 - 3 } Variable & $N$ & Percentage \\
\hline Numbness & 14 & 93 \\
Muscle weakness & 8 & 53 \\
Loss of balance & 8 & 53 \\
Generalized weakness & 6 & 40 \\
Short-term memory loss & 2 & 13 \\
Trouble concentrating & 2 & 13 \\
Lack of coordination & 2 & 13 \\
Loss of depth perception & 1 & 7 \\
\hline
\end{tabular}

Risk of injury. Near or actual injuries were reported by almost $50 \%$ of patients, and were reported to result primarily from non-painful symptoms. A patient with breast cancer reported having to go to the emergency room because of loss of balance and 
falling twice at home, "I hit my right arm and cut the heck out of it on the foot of the bed; I haven't figured out how yet... my arm so bad ...but then the second time, I fell forward and hit my chest of drawers and it blackened my eye, my cheek and my nose, well, in fact, it cracked my nose."Another said "I looked down at my toes one day and I had a purple toe and I didn't know how I had a purple toe; I didn't remember dropping (anything on it), obviously I dropped something pretty hard and I literally had a purple toe for it's going on three months now and you don't feel it; I mean, I don't feel the pain."

A patient in her forties reported, "I always take baths and slowly, I noticed I was having a hard time getting myself to stand up to get out of the bathtub. I cannot get out of the bathtub by myself. Sometimes I do it, but I know one of these days I'm going to end up probably knocking my teeth out or knocking my face off or something because I'm trying to push on the side of the tub to push up and it's slippery." Forty percent of patients who were completely ambulatory before starting chemotherapy required assistive devices such as a cane or walker because of neuropathic symptoms. One patient with lung cancer reported, "I don't have any feeling so I lose my balance, but I get a lot of weakness in my legs and my thighs. Sometimes when I'm on the cane, sometimes I'll lose my balance and... like my leg will give way, just for a short period."

Painful symptoms. The most commonly reported painful symptoms were tingling, burning, muscle aches, and sensitivity to cold (Table 3). These symptoms were reported in at least $33 \%$ of patients. Although two-thirds of participants $(n=10)$ used the word "pain" to describe their experience, one-third of participants did not use the word "pain" 
$(n=5)$. Patients described a host of unpleasant sensations including feelings that their feet were "ice cold", they were "walking on hot coals", "walking on a rock on the bottom of your feet" or with "sandpaper on the bottom of your feet". They often had trouble describing these symptoms succinctly and struggled to put words to their experience. One female patient described her experience in the following way: "Yeah, it feels like...I think I've got something crawling on me; I reach down and there's nothing". One female under treatment with docetaxel for breast cancer responded to a question about how neuropathic symptoms had affected her life with the following: "With my husband and I, intimately, you know, as far as my sex life I'm hurting, I just...I hurt and my legs really hurt and it's been very dragging on my quality of life very much."

Patients who did have pain described it using a variety of terms. Although burning was the adjective most often used to describe pain, several other descriptors including sharp, shooting, pins and needles, muscle aches, soreness, trampling, stabbing, electriclike and pressure were used. The location of pain was generally the upper and/or lower extremities, but two participants described jaw pain and two participants described joint pain. Both of the patients who described jaw pain had received oxaliplatin. Both of the patients who described joint pain had received taxanes. Patients occasionally reported muscle tremors. Sensitivity to cold temperatures was not limited to patients receiving oxaliplatin but also occurred in patients receiving other neurotoxic chemotherapies. No specific pattern in timing of painful symptoms was identified. 
Table 3. Frequencies and percentages for painful symptoms.

\begin{tabular}{lcc}
\hline Variable & $N$ & Percentage \\
\hline Tingling & 7 & 47 \\
Cold Sensitivity & 7 & 47 \\
Pain & 10 & 67 \\
Burning & 6 & 40 \\
Muscle aches & 5 & 33 \\
Pins and needles & 4 & 27 \\
Soreness & 3 & 20 \\
Tremors & 3 & 20 \\
Jaw Pain & 2 & 13 \\
Joint pain & 2 & 13 \\
Sharp pain & 2 & 13 \\
Shooting pain & 2 & 13 \\
Trampling pain & 1 & 7 \\
Stabbing pain & 1 & 7 \\
Electric like pain & 1 & 7 \\
Pressure & 1 & 7 \\
\hline
\end{tabular}

Interference with Activities. Patients described a variety of ways in which neuropathic symptoms interfered with manual dexterity, general activities, activities of daily living, driving, writing, picking up things, work, sleep, walking, hobbies, household duties, and exercise (Table 4). One patient, a physician who finished chemotherapy over two years ago, described how neuropathies have interfered with his ability to perform his job. "Both the combination neuropathy and the sciatic nerve problem forced me to close my medical practice. When I finished with the chemo, the neuropathy really prevented me from doing the endoscopic procedures that I had done previously or standing for long periods of time. In my specialty of gastroenterology, you have to stand for hours at a time. I had to close the office. So for me, that's been a major personal effect. It's changed my whole life." Unfortunately, he also reported his neuropathic symptoms had worsened, rather than regressed, since he completed his chemotherapy. 
Many described how neuropathic symptoms interfere with their usual activity. One female who had received thalidomide for multiple myeloma spoke of it in this way: "I just get discouraged and down and...cause I was always a doer, person that was on the go doing things and I can't...can't do any of that." Another patient, an attorney who also is disabled as a result of advanced lung cancer reported, "I didn't realize how much I used my hands. My writing is shot; it was never very good to begin with and even typing, I've even quit fooling around with e-mail because it's just too painful. I'm not driving very much anymore; driving feels weird too. I can barely feel the pedals." Another male patient who previously enjoyed restoring antique automobiles was distraught over the fact that he no longer has the manual dexterity to work on his cars. When asked about how neuropathic symptoms have interfered with his ability to participate in this activity he reports "I can wax them; that's about it, as far as working on them, no. I get my son to do that. It's because my fingers are numb and I mean, if I go to pick up a screwdriver; I don't know it's there; I have to watch and make sure I have it; it ain't like you can feel it when you pick it up. And when I walk, it just feels like I'm dragging dead feet with me and if my shoe comes off, I don't know it until it's off, so I can't do things. I have to depend on my son if I need to change an alternator or something; I have to get him to do it but I want to do it cause I want to get my hands greasy. I want to pull a motor out or something, put another motor in, I can't do it. I hate to ask people for help. I have been around trucks and cars all my life. So now the brakes are put on." 


\section{Instrument Development}

The preliminary version of the CIPNAT (Appendix B) was developed based upon the above findings. The overall design of the instrument, as previously mentioned, is based upon the theoretical perspective that evaluation of symptoms should include character, timing, intensity and distress (Lenz, Pugh, Milligan, Gift, \& Suppe, 1997; Lenz, Suppe, Gift, Pugh, \& Milligan, 1995). Character is evaluated initially by asking participants whether they are experiencing a specific neuropathic symptom. Choices are limited to "yes" or "no". If they respond "yes" they are directed to answer additional items evaluating timing, intensity, and distress. If they answer "no", they skip items pertaining to that symptom and move to the next symptom. This approach was taken to minimize burden to patients by not having them respond to questions that are not applicable to their individual symptoms.

The symptom interference items ask patients to measure on a numeric rating scale, how neuropathic symptoms are interfering with their ability to carry out usual activities. Participants will only complete symptom interference items if they reported the presence of at least one neuropathic symptom. Specific items selected for inclusion are discussed in the following sections.

Because participants in this study reported numerous injuries or potential injuries as a result of neuropathic symptoms, the author chose to include assessment of injury related to symptoms as part of the instrument. Participants are asked if they have had any injuries as a result of neuropathic symptoms. Possible responses to this item are either "yes" or "no". 
Painful symptoms. Among painful symptoms, burning pain, tingling, cold sensitivity, and muscle aches were the most frequently reported. These four most prevalent symptoms occurred in at least $33 \%$ of participants and therefore were included as items on the preliminary version of the CIPNAT. Because there was a great degree of heterogeneity in descriptions of pain, instead of asking about "burning" pain specifically, a more general item asking whether patients have developed pain since starting chemo and allowing them to select from a list of reported pain descriptors including burning, sharp, shooting, electric-like, stabbing, pins and needles, sore, or other was more appropriate. Next to the word other, a line for patients to include their own description was included. This allows for a more thorough assessment of painful neuropathic symptoms. Tingling in the hands is evaluated separately from tingling in the feet. Previously developed instruments for CIPN have also evaluated tingling in the feet and tingling in the hands separately (Almadrones et al., 2004; Huang et al., 2007) and factor analysis in earlier studies suggests that CIPN may be comprised of two factors; hand symptoms and foot symptoms (Almadrones et al., 2004). In addition, tingling and numbness are believed to begin in the most distal parts of the extremities (fingertips and toes) and progressively involve more of the extremities. Involvement of more of the extremities correlates with more severe CIPN (Ocean \& Vahdat, 2004; Visovsky, 2003; Wilkes, 2007). Accordingly, evaluation of tingling and numbness by the CIPNAT includes assessment of how much of the upper and lower extremities are involved. 
Non-painful symptoms. Among non-painful symptoms, numbness, muscle weakness, loss of balance, and generalized weakness were reported by at least $33 \%$ of patients and were included as items for the CIPNAT. Separate items assess foot and hand numbness, again based upon the results of prior studies (Almadrones et al., 2004).

Symptom interference. Participants in this study reported interference with 13 aspects of daily life including a general category for activities of daily living (Table 4). Most of the categories of interference involved physical outcomes and were included as items on the CIPNAT. Because the purpose of the CIPNAT is to evaluate specific physical symptoms of CIPN, general activity, hobbies and relationships were not initially included. These were thought to represent psychosocial concepts that do not fit well with the other items. The resulting 10 item symptom interference scale asks participants to evaluate how much neuropathic symptoms as a whole interfere with their ability to: walk, pick up things, drive, do household chores, work, use their fingers/hands to do things (manual dexterity), write, exercise, sleep, and engage in sexual activity. If patients do not normally engage in the selected activity, the instructions direct them to mark zero (not at all).

Table 4. Frequencies and percentages for interference with usual activities

\begin{tabular}{llc}
\hline Interference with & $N$ & Percentage \\
\hline Activities of daily living & 8 & 53 \\
Walking & 7 & 47 \\
Picking up things & 6 & 40 \\
Driving & 5 & 33 \\
Hobbies & 5 & 33 \\
Relationships & 4 & 27 \\
Household chores & 3 & 20 \\
Work & 3 & 20 \\
Manual Dexterity & 3 & 20 \\
Writing & 2 & 13 \\
Exercise & 1 & 7 \\
Sleep & 1 & 7 \\
Sexual activity & 1 & 7 \\
\hline
\end{tabular}




\section{Discussion}

This phase I study highlights which neuropathic symptoms are reported most frequently in cancer patients receiving chemotherapy, how patients describe their symptoms, and how these symptoms affect their daily lives. Patients are viewed as experts on their own experience with CIPN. The resulting CIPNAT was developed from the responses of participants. This study provides critical information about which neuropathic symptoms are frequently reported by patients as well as what terminology they use to describe these symptoms and how symptoms affect their daily lives.

In spite of the usefulness of this study, several limitations exist. The sample size was small, limiting the generalizability of the results. The sample was selected from a single geographic location from a site location where patients tend to be of above average education and socioeconomic status, although no data on these variables were collected. Few minorities participated in this study. Results may differ based on racial or ethnic variations. Perhaps the most significant limitation is the possibility of bias in the study. The dual role of the researcher of health care professional and researcher may have caused patients to withhold information that may influence healthcare decisions. It is also possible that the pre-established rapport between participants and researcher allowed for more honest and straightforward discussion. The selection of participants was not random but purposive and therefore the experience of these patients may differ from those in a randomly drawn sample.

Several of the patients had completed chemotherapy up to three years ago and still were experiencing neuropathic symptoms. Because of the significant lapse in time from 
onset of symptoms, their descriptions may not be entirely reflective of their actual experience. In addition, no comprehensive assessment of neuropathic symptoms was conducted. Patients were free to report any neuropathic symptoms they chose to discuss in any amount of detail they were comfortable with. They may have experienced additional neuropathic symptoms that were not discussed in the interview. Conversely, patients may have reported symptoms during the interview that do not have an underlying neuropathic etiology. Ability to differentiate cause of symptoms varies from person to person. The opportunity to speak of their symptom experience in an open ended discussion may have caused some individuals to report symptoms that were distressing but not necessarily of neuropathic origin. To compensate for this limitation, the selection of items for the CIPNAT was limited to symptoms that had been previously documented in earlier research as having an underlying neuropathic cause. 


\section{Chapter Four}

Phase II Method, Results and Discussion

This chapter discusses the second phase of instrument development and testing, review by a panel of experts. Review by a panel of experts is helpful in evaluating content validity and implementing any necessary changes to the instrument (Waltz, Strickland \& Lenz, 2005). This chapter discusses methods and results of this Phase II study. Scoring of the CIPNAT also is discussed.

\section{Method}

The second phase of instrument development was to evaluate content validity of the first draft of the CIPNAT. This was accomplished by consultation with a panel of five experts. Experts evaluated whether items developed from the results of the phase I study accurately represent the characteristics of CIPN.

Consultants. A neurologist, a medical oncologist, two $\mathrm{PhD}$ prepared registered nurses and an oncology certified registered nurse made up the panel of experts. Members of the panel of experts were selected based on their knowledge and experience with CIPN. Requirements for selection in the panel of experts were direct and consistent involvement in the care of persons with CIPN or publication on this topic. 
The consultants are described here. Ron D. Schiff, MD, PhD is a board certified medical oncologist in Tampa. He has extensive knowledge of chemotherapy and its related side effects. Before entering private practice, he was a professor at the University of South Carolina School of Medicine. He completed his residency in oncology at Memorial Sloan Kettering Cancer Center in New York.

Leon Prockop, MD is a board certified neurologist in Tampa, Florida. He is a professor at the University of South Florida College of Medicine. He is a graduate of Princeton University and the University of Pennsylvania, College of Medicine. He has authored at least 73 peer reviewed journal articles and 61 book chapters on a variety of subjects within his specialty.

Constance Visovsky, $\mathrm{PhD}$, ARNP is a nurse practitioner and associate professor at the University of Nebraska. She has published at least five articles since 2003 on CIPN. She served on an Oncology Nursing Society taskforce that evaluated the literature pertaining to CIPN and made recommendations for nursing management (ONS, 2005). Marie Bakitas, DNSc, ARNP, FAAN is an assistant professor at Dartmouth Medical School and Nurse Practitioner at Dartmouth-Hitchcock Medical Center in Hanover, New Hampshire. She is also a postdoctoral fellow at the Yale University Medical Center in New Haven, Connecticut. She published a qualitative study on CIPN entitled "Background noise: the experience of chemotherapy-induced peripheral neuropathy" (Bakitas, 2007).

Patricia Friedel, RN, BSN, OCN is a registered nurse in the infusion center at Moffitt Cancer Center. She received her bachelor's degree from Kent State University in 
1989. She has been a chemotherapy nurse in the Moffitt Infusion Center for five years and is currently working toward her Master's degree at the University of Tampa.

Procedures. An evaluation tool was designed to help the consultants provide feedback on the CIPNAT. The Content Validity Assessment tool provided consultants with operational definitions necessary to evaluate the items as well as a list of items from the CIPNAT. Each expert was provided with the Content Validity Assessment tool and asked to rate individual items for relevance to either CIPN as a whole or to specific neuropathic symptoms. The rating system was as follows: $1=$ the item is not relevant to CIPN or to a specific neuropathic symptom, $2=$ the item is somewhat relevant to CIPN or to a specific neuropathic symptom, $3=$ the item is quite relevant to CIPN or to a specific neuropathic symptom or $4=$ the item is very relevant to CIPN or to a specific neuropathic symptom (Grant \& Davis, 1997). The experts were also asked to evaluate how comprehensive the CIPNAT is in its approach to evaluating symptoms, how appropriate the items are for patients in terms of how easy it is to understand each item, and whether items are written at an appropriate grade level. There were also areas for consultants to write in additional comments.

Data obtained from the panel of experts was analyzed by calculating a content validity index (CVI) on every item and on the entire instrument. The CVI is defined as the proportion of items given a rating of quite relevant or very relevant by the experts. Items with a CVI of .80 were evaluated for deletion or revision. This was a conservative approach because a CVI of 0.8 is considered acceptable in most cases (Wynd, Schmidt, \& Schaefer, 2003). The CVI for the entire instrument was calculated as a mean CVI for 
all items. Comments and suggestions by the experts were taken into account and appropriate items were added or deleted from the instrument as needed.

Results

CVI's for individual items ranged from 0.8-1.0. Twenty-two of 85 items had CVI's of .80 and 63 items had CVI's of 1.0. The CVI for the entire instrument was 0.95 . Items related to muscle or joint aches and pain were revised. Items related to generalized weakness were eliminated and items related to muscle weakness were revised. Additional items related to interference with "relationships", "hobbies or leisure activities", and "enjoyment of life" were added. Items pertaining to frequency of symptoms were revised. Minor changes in wording were made to several additional items for the purpose of clarity. Items containing terms that were thought to be "too medical" were also revised. Items that were redundant or not critical to the evaluation of CIPN were eliminated based on feedback from experts that the instrument was too lengthy. The resulting version of the CIPNAT contains a total of 69 items evaluating nine specific neuropathic symptoms, neuropathic interference with usual activities, and injuries resulting from neuropathic symptoms. The instructions for patients were revised to encourage the patient to allow them to ask someone to read the questions to them and record their responses. Instructions also encouraged rest during completion as necessary. Scoring

The original version of the CIPNAT consisted of 85 items. Revisions to the instrument following recommendations by the panel of experts and analysis of the content validity index resulted in a 69 item instrument. Forty-five items assess symptom 
occurrence, severity, intensity and frequency. Fourteen of the 69 items are descriptive data which is useful for understanding the entire neuropathic symptom experience but do not contribute to the total score. These items make up the symptom experience scale. Scores for each scored item range from 0-10. Total symptom experience scores range from 0-400 (Table 5). If items are skipped because they are not applicable, a zero score on that item is assigned. Scores on each of the 15 items evaluating symptom interference range $0-10$. Total interference scale scores range from $0-150$. Scores on the symptom experience scale are added to the interference score to form a total score.

\section{Discussion}

Evaluation of the CIPNAT by a panel of five experts demonstrated content validity of the instrument. Changes were made to the CIPNAT based on the recommendations of the panel of experts. The suggestions made by the experts resulted in a briefer yet more comprehensive instrument. In future studies, the researcher plans to develop an electronic version of the CIPNAT. An electronic format may be more applicable to this type of instrument which is designed to be tailored to each patient's specific symptoms. In scoring this instrument, the author chose to sum scores related to symptom prevalence, intensity, severity, and distress. There is some question as to whether these scores can be added together to form a symptom experience score or whether subscores should be calculated for each dimension of the symptom experience. Data from the CIPNAT will be used to test the Theory of Unpleasant Symptoms. Doing so may provide useful information as to whether intensity, frequency, and distress have a summative effect or need to be examined separately. 
Table 5. Scoring guide.

\begin{tabular}{|c|c|c|c|}
\hline & Description & Possible Points & Items Contributing to Total Score \\
\hline \multicolumn{4}{|c|}{ Symptom Experience Scale } \\
\hline \multirow[t]{5}{*}{1} & numbness hands & 50 & 1a (10 points) \\
\hline & & & $1 \mathrm{~b}(10$ points $)(2.5 \mathrm{x} 4)$ \\
\hline & & & $1 \mathrm{c}(10$ points $)$ \\
\hline & & & $1 \mathrm{~d}$ (10 points) \\
\hline & & & 1e (10 points) \\
\hline \multirow[t]{5}{*}{2} & numbness feet & 50 & $2 \mathrm{a}$ (10 points) \\
\hline & & & $2 \mathrm{~b}(10$ points $)(2 \times 5)$ \\
\hline & & & $2 \mathrm{c}(10$ points $)$ \\
\hline & & & $2 \mathrm{~d}$ (10 points) \\
\hline & & & $2 \mathrm{e}(10$ points $)$ \\
\hline \multirow[t]{5}{*}{3} & tingling hands & 50 & $3 a$ (10 points) \\
\hline & & & $3 \mathrm{~b}(10$ points $)(2.5 \mathrm{x} 4)$ \\
\hline & & & $3 \mathrm{c}$ (10 points) \\
\hline & & & $3 \mathrm{~d}$ (10 points) \\
\hline & & & $3 \mathrm{e}$ (10 points) \\
\hline \multirow[t]{5}{*}{4} & tingling feet & 50 & $4 \mathrm{a}$ (10 points) \\
\hline & & & $4 \mathrm{~b}(10$ points $)(2 \times 5)$ \\
\hline & & & $4 \mathrm{c}$ (10 points) \\
\hline & & & $4 \mathrm{~d}$ (10 points) \\
\hline & & & 4e (10 points) \\
\hline \multirow[t]{4}{*}{5} & cold sensitivity & 40 & $5 a$ (10 points) \\
\hline & & & $5 \mathrm{c}(10$ points $)$ \\
\hline & & & $5 \mathrm{~d}$ (10 points) \\
\hline & & & $5 \mathrm{e}$ (10 points) \\
\hline \multirow[t]{4}{*}{6} & nerve pain & 40 & $6 a$ (10 points) \\
\hline & & & $6 \mathrm{c}$ (10 points) \\
\hline & & & $6 \mathrm{~d}$ (10 points) \\
\hline & & & $6 \mathrm{e}$ (10 points) \\
\hline \multirow[t]{4}{*}{7} & myalgias/arthralgias & 40 & $7 \mathrm{a}(10$ points $)$ \\
\hline & & & 7c (10 points) \\
\hline & & & $7 \mathrm{~d}$ (10 points) \\
\hline & & & 7e (10 points) \\
\hline \multirow[t]{4}{*}{8} & muscle weakness & 40 & $8 \mathrm{a}(10$ points $)$ \\
\hline & & & $8 \mathrm{c}$ (10 points) \\
\hline & & & 8d (10 points) \\
\hline & & & $8 \mathrm{e}(10$ points $)$ \\
\hline \multirow[t]{4}{*}{9} & loss of balance & 40 & 9a (10 points) \\
\hline & & & $9 \mathrm{~b}$ (10 points) \\
\hline & & & $9 \mathrm{c}$ (10 points) \\
\hline & & & $9 \mathrm{~d}$ (10 points) \\
\hline Score & & $0-400$ & \\
\hline $\begin{array}{l}\text { Interference } \\
\text { Scale }\end{array}$ & Various activities & $0-150$ & 10a-10o (10 points each) \\
\hline
\end{tabular}




\section{Chapter Five}

Phase III Method, Results and Discussion

Phase III methods for assessing reliability and construct validity of the CIPNAT are presented in this chapter. Results of this study are then presented, followed by discussion. Discussion includes importance to nursing and future use of the CIPNAT. Method

The phase III study was an exploratory, descriptive study designed to evaluate validity and reliability of the revised CIPNAT. This phase of the study was conducted at the Moffitt Cancer Center and the private medical oncology practice of Ron D. Schiff, $\mathrm{MD}, \mathrm{PhD}$ and Egberto Zayas, MD. For this phase of the study, approval from both the Moffitt Scientific Review Board and the University of South Florida IRB was obtained. Permission to conduct the study at their practice was obtained from Dr. Schiff and Dr. Zayas. All data were collected between August 2008 and October 2008.

A convenience sample of 167 outpatients currently undergoing chemotherapy for cancer participated in this study. Eligibility criteria included: 1) a diagnosis of cancer; 2) able to speak and understand English; and 3) between 18 and 90 years of age. One hundred-twenty seven patients had received at least one chemotherapy treatment with paclitaxel, docetaxel, cisplatin, or oxaliplatin within the last 6 months. A comparison group of 40 neurotoxic chemotherapy naive patients, who were receiving other types of 
chemotherapy were recruited. Patients were excluded if they had preexisting diabetic neuropathy or if they have known dementia or psychiatric illness.

\section{Instruments}

Functional Assessment of Cancer Therapy/Gynecologic Oncology Group Neurotoxicity Scale. The Functional Assessment of Cancer Therapy/Gynecologic Oncology Group Neurotoxicity Scale (FACT/GOG-Ntx) (Appendix A) is an 11 item Likert-type scale assessing neuropathic symptoms (Appendix A). Participants are asked to rate the extent to which they agree with given experiences over the past seven days. Possible responses range from $0=$ not at all to $4=$ very much. Higher scores correspond with a higher degree of neuropathic symptoms.

Evidence of validity was provided by Calhoun and colleagues (2003) who found significant correlations between the FACT/GOG-Ntx and neurological examination evaluating sensory symptoms, pin sensibility, strength, deep tendon reflexes, vibration sensibility, and nerve conduction. Significant differences in scores from chemotherapy naïve individuals and those with known chemotherapy induced peripheral neuropathy demonstrated construct validity. Cronbach's alphas ranged from .78 - .88.

Chemotherapy Induced Peripheral Neuropathy Assessment Tool. The Chemotherapy Induced Peripheral Neuropathy Assessment Tool (CIPNAT) (Appendix B) is the subject of this study. As discussed, it was developed from interviews with 15 patients with chemotherapy induced peripheral neuropathy. The instrument assesses the multi-dimensional characteristics of CIPN including presence of neuropathic symptoms, symptom severity, symptom distress and timing of symptoms (Lenz et al., 1997; Lenz et 
al., 1995). It also assesses neuropathic symptom interference with normal activities and patient perception of progression of neuropathic symptoms.

The initial items for the instrument were selected based on which symptoms were most frequently reported by patients in the phase I study. Symptoms that were reported by at least $33 \%$ of patients were included. These symptoms make up the symptom experience scale. Nine neuropathic symptoms including; numbness in the hands, numbness in the feet, tingling in the hands, tingling in the feet, sensitivity to cold temperatures, nerve pain, muscle/joint aches, muscle weakness and loss of balance are assessed. Higher scores on the symptom experience scale correspond with higher degrees of CIPN. The second aspect of the CIPNAT is the interference scale. Items in the interference scale were reported by patients in the qualitative study (Table 4). Higher scores on this scale correspond with greater neuropathic interference with usual activities.

Demographic data form. A demographic data form (Appendix C) was developed for this study. It includes age, gender, race/ethnicity, income, education, marital status, income, years of formal education, employment status, type of cancer, type of chemotherapy, number of cycles of chemotherapy, and cumulative dose of neurotoxic agent. Chemotherapy information was completed by the researcher with information obtained from the medical record.

\section{Procedures}

Approvals from both the Moffitt Cancer Center Scientific Review Committee and the practice of Dr. Schiff and Dr. Zayas were obtained. Support for the recruitment of participants from the Moffitt Infusion Center was obtained from the Clinical Operations 
Manager of the Infusion Center. Following these approvals, approval from the University of South Florida IRB was obtained. Patients who were appropriate for the study were identified by referring to a list of current patients which is kept in the nursing station in the infusion center. This list describes what chemotherapy regimen patients are receiving that day. Patients who were receiving paclitaxel, docetaxel, oxaliplatin, or cisplatin were approached to determine if they met eligibility criteria and determine their interest in participation. Patients who were receiving non-neurotoxic chemotherapy for any type of cancer were also identified through the list of patients kept in the infusion center.

A convenience sample of 127 patients who had received at least one course of chemotherapy with paclitaxel, docetaxel, cisplatin, or oxaliplatin were recruited from the infusion centers. These patients were considered at high risk for chemotherapy induced peripheral neuropathy. An additional 40 cancer patients who had not received chemotherapy with paclitaxel, docetaxel, cisplatin, or oxaliplatin, and who were at low risk of chemotherapy induced peripheral neuropathy, were recruited. Patients who were receiving their first treatment with paclitaxel, docetaxel, cisplatin, or oxaliplatin on the day of the interview and had never received any other forms of neurotoxic chemotherapy were included in this group of 40 patients because they had not yet had time to develop neurotoxicities.

Patients were approached by the researcher and informed of study requirements and risks and benefits of taking part in the study. If they agreed to participate, informed consent was obtained. The researcher asked the participants all items on the demographic form, the CIPNAT, and the Functional Assessment of Cancer Therapy/Gynecologic 
Oncology Group-Neurotoxicity questionnaire (FACT\&GOG-Ntx) and recorded their verbal responses on a questionnaire form. Questionnaires were orally administered at the time of consent while patients were in their private chemotherapy suites. If patients preferred to complete the questionnaires independently, they were permitted to do so. Thirty of the participants on neurotoxic chemotherapy were asked to complete a second CIPNAT for test-retest reliability over the phone 24-72 hours after chemotherapy or in person if they would be coming back to the site during that time. If they agreed, they were asked for a phone number where they could be contacted and an appointment time for the phone interview was mutually agreed upon. The provided phone number was kept on a separate log which contained only their subject number, phone number, appointment date and time, and whether the investigator was able to contact them for the interview. Phone numbers were deleted from the log, once the second set of questionnaires had been completed. If the researcher was unable to contact the participant at the provided phone number within seven days of completing the first set of questionnaires, the phone number was deleted from the log and no further attempts to contact that participant were made.

Evaluating validity. Validity testing is necessary to ensure that an instrument measures what it is intended to measure (Waltz, Strickland, \& Lenz, 2005). Content validity was assessed in the phase II study. In this phase III study, three techniques were used to evaluate construct validity. First, the instrument was administered to a group of cancer patients at high risk for neuropathic symptoms as well as to a comparison group of cancer patients who were at low risk for neuropathic symptoms. The results were compared using t-tests to determine whether scores of the two groups differed 
significantly on total scores. Significant differences between one group expected to have high scores on the CIPNAT and another group expected to have low scores on the CIPNAT provided evidence of validity.

Correlation with a measure of the same or similar concept was also provided evidence of validity. The questionnaire was administered to a group of cancer patients along with an instrument purported to measure similar concepts, the neurotoxicity scale of the FACT\&GOG-Ntx (Huang et al., 2007). Pearson's correlations were used to evaluate correlations between the CIPNAT and the FACT/GOG-Ntx and provided additional evidence of construct validity. Moderate to high correlations between the CIPNAT and the neurotoxicity scale of the FACT\&GOG-Ntx were expected.

Confirmatory factor analysis was performed using data from the 127 patients who have received neurotoxic chemotherapy to confirm the factor structure and provide further evidence of construct validity. It was hypothesized that factor analysis of the symptom experience scale would indicate that the CIPNAT symptom experience scale measures two underlying latent constructs: painful symptoms and non-painful symptoms. An alternative hypothesis was that the factor analysis would confirm two latent constructs of sensory symptoms and motor symptoms. The interference with usual activities scale was hypothesized to measure a single underlying latent construct, interference with daily activities.

Evaluating reliability. Reliability refers to how consistently an instrument assigns scores to subjects (Waltz et al., 2005). Reliability for the instrument was evaluated by using the test-retest procedure. Neuropathy is conceptualized as relatively stable over a 
short period of time for participants actively receiving chemotherapy and for a longer time frame for persons not on chemotherapy. Scores from test to retest are expected to be highly correlated. Because some neuropathic symptoms may be most noticeable in the first 24-72 hours after chemotherapy, patients who are getting neurotoxic chemotherapy completed the second CIPNAT by telephone approximately 24-72 hours after the first set of questionnaires was administered. If the patients were to be returning to the clinic within 24-72 hours, the researcher met them there for the purpose of having them complete the second set of questionnaires. Patients who had difficulty writing due to CIPN had each item read to them so they could respond verbally, with the researcher recording their responses. Internal consistency reliability was evaluated using Cronbach's alpha. Because the instrument was designed to measure more than one concept, alpha levels were determined for each scale, subscale, and for the entire instrument.

Data analysis. Data were analyzed using Statistical Package for the Social Sciences (SPSS) version 16. Factor analysis were conducted using both SPSS v. 16 and LISREL 8.0. The data was entered into SPSS and examined for data entry errors. Descriptive statistics were analyzed. Exploratory factor analysis was conducted in SPSS and confirmatory factor analysis was conducted in LISREL for the purpose of identifying the factor structure for the symptom experience scale and the symptom interference scale. Correlations and differences between means also were used to evaluate validity. Correlations were used to evaluate test-retest reliability. Internal consistency reliability of the symptom experience scale and the interference scale were evaluated using Cronbach's alpha. Standardized alpha coefficients allow for comparison between data 
collected using two different instruments using the same group of participants

(Alsawalmeh \& Feldt, 2000). Standardized alpha coefficients between the CIPNAT and FACT-Ntx were compared.

Results

Demographics. There were 127 participants in the group receiving neurotoxic chemotherapy and 40 participants in the comparison group. Ages ranged from 19 to 80 with a mean age of 58. Sixty percent of participants were female and $40 \%$ were male. The majority were Caucasian, married and either retired or disabled (Table 6). Twentyseven percent continued to work on a part-time or full-time basis. Participants had a diverse range of incomes and educational backgrounds. Almost all participants had solid tumors. Breast, lung, or colon cancers were the most frequently occurring sites. Most had stage 3 or 4 disease. From the patients in the group who were on treatment with neurotoxic chemotherapy, approximately $30 \%$ were receiving paclitaxel, $31 \%$ were receiving docetaxel, $27 \%$ were receiving oxaliplatin and $13 \%$ were receiving cisplatin. They had received from 1 to 30 treatments with a mean of 5.2 treatments and had received an average of $500 \mathrm{mg} / \mathrm{m}^{2}$ of neurotoxic drug.

Descriptive symptom data. In the group receiving neurotoxic chemotherapy, cold sensitivity and muscle/joint aches were the symptoms which were most often reported, followed by numbness in the hands, numbness in the feet, trouble with balance, muscle weakness, tingling in the hands, tingling in the feet and nerve pain. Nerve pain was the most severe symptom. Muscle/joint aches were the most distressing symptom. Numbness in the feet was the symptom that patients reported as most constant, or frequently 
occurring (Table 7). It should be noted that $14.17 \%(n=18)$ of patients receiving neurotoxic chemotherapy reported no neurotoxicity symptoms.

Frequencies for the symptom interference items show that neuropathic symptoms interfered with enjoyment of life, sleep and chores in more than half of participants being treated with neurotoxic chemotherapy. Neuropathic symptoms interfered with walking, exercise and hobbies in $48 \%$ of participants. Neuropathic symptoms also interfered with working, picking up objects, writing, holding onto objects, relationships, driving, dressing and sexual activity(Table 8).

Twenty- two percent $(n=28)$ had experienced injuries as a result of neuropathic symptoms. The majority of injuries were falls $(n=21)$ or injuries caused by bumping into things $(n=4)$. Pulled back muscle $(n=1)$, poor wound healing $(n=1)$ and acute hypersensitivity reaction $(n=1)$ were reported but were not thought to be related to neuropathic symptoms. Ninety two percent $(n=23)$ of patients reporting falls or bumping into things also reported loss of balance. 
Table 6. Frequencies and Percentages of Demographic Data

\begin{tabular}{|c|c|c|c|c|c|}
\hline \multirow{2}{*}{ Demographic Data } & & \multicolumn{2}{|c|}{ Neurotoxic chemo $n=127$} & \multicolumn{2}{|c|}{ comparison $n=40$} \\
\hline & & Frequency & Percent & Frequency & Percent \\
\hline \multirow[t]{5}{*}{ Marital status } & single & 14 & 11 & 4 & 10 \\
\hline & married & 82 & 64.6 & 24 & 60 \\
\hline & divorced & 16 & 12.6 & 5 & 12.5 \\
\hline & separated & 3 & 2.4 & 1 & 2.5 \\
\hline & widowed & 12 & 9.4 & 6 & 15 \\
\hline \multirow[t]{6}{*}{ Income } & less than $25 \mathrm{~K}$ & 30 & 23.6 & 7 & 17.5 \\
\hline & $25-50 \mathrm{~K}$ & 26 & 20.5 & 10 & 25 \\
\hline & $50-75 \mathrm{~K}$ & 19 & 15 & 10 & 25 \\
\hline & over $75 \mathrm{~K}$ & 26 & 20.5 & 9 & 22.5 \\
\hline & prefer not to answer & 26 & 20.5 & 3 & 7.5 \\
\hline & Missing data & 0 & 0 & 1 & 2.5 \\
\hline \multirow[t]{5}{*}{ Education } & $8-12$ years & 43 & 33.9 & 18 & 45 \\
\hline & $13-14$ years & 36 & 28.3 & 11 & 27.5 \\
\hline & $15-16$ years & 33 & 26.0 & 7 & 17.5 \\
\hline & $17-23$ years & 13 & 10.4 & 4 & 10 \\
\hline & Missing Data & 2 & 1.6 & 0 & 0 \\
\hline \multirow[t]{5}{*}{ Race/Ethnicity } & $\begin{array}{l}\text { American Indian/Alaskan } \\
\text { Native }\end{array}$ & 2 & 1.6 & 1 & 2.5 \\
\hline & Asian & 2 & 1.6 & 1 & 2.5 \\
\hline & African American & 7 & 5.5 & 2 & 5 \\
\hline & Hispanic & 8 & 6.3 & 2 & 5 \\
\hline & White/Caucasian & 108 & 85 & 34 & 85 \\
\hline \multirow[t]{8}{*}{ Employment } & full time & 28 & 22 & 8 & 20 \\
\hline & part time & 5 & 3.9 & 3 & 7.5 \\
\hline & on leave of absence & 15 & 11.8 & 2 & 5 \\
\hline & retired & 44 & 34.6 & 16 & 40 \\
\hline & disabled & 25 & 19.7 & 10 & 25 \\
\hline & full-time student & 1 & 0.8 & 0 & 0 \\
\hline & unemployed & 7 & 5.5 & 1 & 2.5 \\
\hline & self-employed & 2 & 1.6 & 0 & 0 \\
\hline \multirow[t]{6}{*}{ Cancer Type } & breast & 26 & 20.5 & 16 & 40 \\
\hline & lung & 20 & 15.7 & 15 & 37.5 \\
\hline & colon & 33 & 26 & 4 & 10 \\
\hline & gynecologic malignancies & 10 & 7.8 & 1 & 2.5 \\
\hline & Other solid tumors & 37 & 29.1 & 3 & 7.5 \\
\hline & Hematologic malignancies & 1 & 0.8 & 0 & 0 \\
\hline \multirow[t]{7}{*}{ Cancer Stage } & 1 & 4 & 3.1 & 2 & 5 \\
\hline & 2 & 15 & 11.8 & 6 & 15 \\
\hline & 3 & 37 & 29.1 & 15 & 37.5 \\
\hline & 4 & 62 & 48.8 & 10 & 25 \\
\hline & Extensive small cell lung & 2 & 1.6 & 4 & 10 \\
\hline & Limited small cell lung & 0 & 0 & 1 & 2.5 \\
\hline & Missing & 7 & 5.5 & 2 & 5 \\
\hline
\end{tabular}


Table 7: Occurrence, severity, intensity, distress and timing of neuropathic symptoms

\begin{tabular}{lllrrrrrr}
\hline & \multicolumn{9}{c}{ mean } & & & & \\
Symptom & $n$ & Percent & severity & SD & mean distress & SD & mean timing & SD \\
\hline Cold Sensitivity & 66 & 51.97 & 5.17 & 2.79 & 4.26 & 3.26 & 5.61 & 2.69 \\
Muscle or joint & 66 & 51.97 & 6.02 & 2.69 & 5.74 & 2.85 & 5.42 & 2.79 \\
aches & & & & & & & & \\
Numb hands & 63 & 49.60 & 4.00 & 2.34 & 3.43 & 2.94 & 6.49 & 3.04 \\
Numb feet & 60 & 47.24 & 4.97 & 3.00 & 4.08 & 3.40 & 7.37 & 3.08 \\
Loss of Balance & 59 & 39.37 & 4.66 & 2.67 & 4.95 & 3.04 & 5.42 & 2.37 \\
Muscle Weakness & 58 & 45.70 & 5.66 & 2.71 & 5.50 & 3.42 & 5.89 & 2.61 \\
Tingling hands & 56 & 44.09 & 4.04 & 2.52 & 3.91 & 3.00 & 6.20 & 3.08 \\
Tingling Feet & 48 & 37.80 & 4.25 & 2.73 & 3.88 & 3.13 & 6.50 & 3.05 \\
Nerve Pain & 29 & 22.83 & 6.48 & 3.03 & 5.69 & 3.26 & 5.28 & 2.66 \\
\hline
\end{tabular}

Table 8: Frequency of interference items

\begin{tabular}{llllll}
\hline Interference item & $n$ & Percent & mean & SD & Range \\
\hline Enjoyment of Life & 71 & 55.90 & 5 & 3 & $1-10$ \\
Sleep & 69 & 54.33 & 6 & 3 & $1-10$ \\
Chores & 65 & 51.18 & 5 & 3 & $1-10$ \\
Hobbies & 61 & 48.03 & 5 & 3 & $1-10$ \\
Walking & 60 & 47.24 & 5 & 3 & $1-10$ \\
Exercise & 60 & 47.24 & 6 & 3 & $1-10$ \\
Working & 47 & 37.00 & 7 & 3 & $1-10$ \\
Picking up objects & 47 & 37.00 & 5 & 3 & $1-10$ \\
Writing & 44 & 34.65 & 5 & 3 & $1-10$ \\
Holding onto objects & 42 & 33.07 & 4 & 2 & $1-10$ \\
Relationships & 40 & 31.50 & 4 & 2 & $1-10$ \\
Driving & 39 & 30.70 & 5 & 3 & $1-10$ \\
Dressing & 38 & 29.92 & 4 & 3 & $1-10$ \\
Injuries & 28 & 22.05 & N/A & N/A & Yes/no \\
Sexual Activity & 26 & 20.47 & 5 & 3 & $1-10$ \\
\hline
\end{tabular}

Validity. Differences between patients expected to have symptoms of neurotoxity and those not expected to have neurotoxicity were compared using t-tests for unequal variances. Independent t-tests for unequal variance were used because Levene's test for equality of variance indicated that variance between the two groups were different (Table 9). Scores on the CIPNAT as well as the FACT-Ntx demonstrated significant difference between these two groups (Table 9). Correlation with a measure of the same concept 
indicated that scores between the CIPNAT and the FACT-Ntx were strongly correlated ( $\mathrm{r}$ $=.73, \mathrm{p}=.000, \mathrm{n}=127)$, supporting construct validity of the CIPNAT.

Table 9. Independent t-tests for CIPNAT and FACT/GOG-Ntx.

\begin{tabular}{|c|c|c|c|c|c|c|c|}
\hline Instrument & group & $\begin{array}{c}\text { Levene's test for } \\
\text { unequal } \\
\text { variance } \\
F\end{array}$ & $P$ & $N$ & Mean & $S D$ & $P$ \\
\hline \multirow[t]{2}{*}{ CIPNAT } & $\begin{array}{l}\text { neurotoxic } \\
\text { chemo }\end{array}$ & 15.55 & .000 & 126 & 142.62 & 103.00 & .000 \\
\hline & comparison & & & 40 & 33.04 & 59.22 & \\
\hline $\begin{array}{l}\text { Symptom experience } \\
\text { scale }\end{array}$ & $\begin{array}{l}\text { neurotoxic } \\
\text { chemo } \\
\text { comparison }\end{array}$ & 23.28 & .000 & $\begin{array}{l}127 \\
40\end{array}$ & $\begin{array}{l}110.86 \\
22.34\end{array}$ & $\begin{array}{l}80.85 \\
40.37\end{array}$ & .000 \\
\hline \multirow[t]{2}{*}{ Interference scale } & $\begin{array}{l}\text { neurotoxic } \\
\text { chemo }\end{array}$ & 11.11 & .000 & 126 & 29.29 & 27.87 & .000 \\
\hline & comparison & & & 40 & 9.95 & 19.98 & \\
\hline FACT/GOG-NTX & $\begin{array}{l}\text { neurotoxic } \\
\text { chemo } \\
\text { comparison }\end{array}$ & 16.35 & .000 & $\begin{array}{l}127 \\
40\end{array}$ & $\begin{array}{l}9.77 \\
3.35\end{array}$ & $\begin{array}{l}7.55 \\
4.00\end{array}$ & .000 \\
\hline
\end{tabular}

Confirmatory factor analysis was based on the covariance matrix and used maximum likelihood estimation. Confirmatory factor analysis of the nine items from the symptom experience scale indicate that the model representing painful and non-painful symptoms had poor goodness of fit (Table 11) and factor loadings on several items were low (Table 10). The model representing sensory and motor symptoms had a better fit (Table 11) and higher factor loadings (Table 10). Numbness in hands, numbness in feet, tingling in hands, tingling in feet, cold sensitivity, and nerve pain loaded on the sensory factor. Muscle or joint aches, muscle weakness, and loss of balance loaded on the motor factor (Table 10). The model explained from fifteen to $56 \%$ of item variance. Evaluation 
of modification indices suggested that error terms in the theta delta matrix were highly correlated, especially for numbness and tingling in the hands and numbness and tingling in the feet, reducing the goodness of fit of the measurement model (Table 11). When error terms for numbness in the hands and tingling in the hands as well as numbness in the feet and tingling in the feet were allowed to correlate, the fit of the model improved.

Table 10. CFA Factor loadings and squared multiple correlations.

\begin{tabular}{|c|c|c|c|}
\hline Symptom & Non-Painful & Painful & $\mathbf{R}^{2}$ \\
\hline Numb Hands & 0.733 & - - & .538 \\
\hline Numb Feet & 0.649 & - - & .421 \\
\hline Cold Sensitivity & 0.364 & - - & .133 \\
\hline Muscle Weakness & 0.341 & - - & .116 \\
\hline Trouble with Balance & 0.340 & - - & .115 \\
\hline Tingling Hands & -- & 0.671 &. .450 \\
\hline Tingling Feet & - - & 0.644 & .414 \\
\hline Nerve Pain & - & 0.367 & .135 \\
\hline Muscle/joint Aches & - - & 0.291 & .085 \\
\hline Symptom & Sensory & Motor & $\mathbf{R}^{2}$ \\
\hline Numb Hands & $0.678^{\circ}$ & - - & .459 \\
\hline Numb Feet & 0.640 & - & .409 \\
\hline Tingling Hands & 0.608 & - - & .370 \\
\hline Tingling Feet & 0.612 & - - & .375 \\
\hline Cold Sensitivity & 0.389 & - - & .152 \\
\hline Nerve Pain & 0.392 & - - & .154 \\
\hline Muscle/joint Aches & - - & 0.504 & .254 \\
\hline Muscle Weakness & - - & 0.631 & .398 \\
\hline Trouble with Balance & - - & 0.746 & .557 \\
\hline Activity & Manual dexterity & $\begin{array}{c}\text { General } \\
\text { activities }\end{array}$ & $\mathbf{R}^{2}$ \\
\hline Dressing & .731 & - - & .534 \\
\hline Writing & .596 & - - & .355 \\
\hline Picking up objects & .873 & - - & .760 \\
\hline Holding onto objects & .844 & - - & .715 \\
\hline Driving & - - & .569 & .321 \\
\hline Working & - - & .579 & .344 \\
\hline Hobbies & -- & .704 & .503 \\
\hline Exercise & - & .728 & .540 \\
\hline Sex & - - & .317 & .103 \\
\hline Sleep & - & .626 & .395 \\
\hline Relationships & - - & .647 & .419 \\
\hline Walking & - - & .774 & .592 \\
\hline Chores & - - & .871 & .748 \\
\hline Enjoyment of life & - & .795 & .645 \\
\hline Injuries & - & .390 & .152 \\
\hline
\end{tabular}


Table 11. Goodness of fit indices.

\begin{tabular}{|c|c|c|c|c|c|c|}
\hline Scale & df & $\mathbf{X}^{2}$ & RMSEA & GFI & CFI & NFI \\
\hline 2 factor model & 26 & 110.28 & .162 & .836 & .764 & .720 \\
\hline Painful/non-painful & & & & & & \\
\hline $\begin{array}{l}2 \text { factor model } \\
\text { sensory/motor }\end{array}$ & 26 & 70.29 & .106 & .90 & .898 & .842 \\
\hline $\begin{array}{l}\text { Symptom experience } \\
2 \text { factor model with } \\
\text { correlated error terms }\end{array}$ & 24 & 47.733 & 086 & .924 & .938 & .884 \\
\hline $\begin{array}{l}\text { Symptom experience } \\
\text { sensory/motor }\end{array}$ & & & & & & \\
\hline $\begin{array}{l}\text { Interference } \\
2 \text { factor solution }\end{array}$ & 89 & 267.68 & .119 & .792 & .928 & .893 \\
\hline $\begin{array}{l}\text { Interference } \\
2 \text { factor solution with } \\
\text { sex and injury removed }\end{array}$ & 64 & 141.90 & .098 & .853 & .957 & .925 \\
\hline
\end{tabular}

Confirmatory factor analysis of the fifteen items from the interference scale also revealed two underlying latent concepts, activities requiring manual dexterity and general activities. Dressing, picking up objects, holding onto objects and writing loaded on the manual dexterity subscale. Walking, driving, working, participating in hobbies, exercising, sexual activity, sleeping, relationships with other people, doing household chores, and enjoyment of life all loaded on the general activities factor. This model explained $32-76 \%$ of item variance with the exception of two items (Table 10). Only $10 \%$ of the variance in sexual activity was explained by the model. About $15 \%$ of the variance in injuries from neuropathic symptoms was explained by the model. When sexual activity and occurrence of neuropathy related injuries were removed from the model, the goodness of fit of the model improved significantly (Table 11). Again, error terms were highly correlated, reducing the goodness of fit of the model. Modification indices suggested that error terms for "work" and "hobbies or leisure activities" as well as for "picking up things" and "holding onto things" were highly correlated. 
Reliability. The CIPNAT and FACT-Ntx were administered to a subset of 30 patients for test-retest reliability. Test-retest scores on the symptom experience scale $(\mathrm{r}=.897, \mathrm{p}=.000)$ and the interference with activities scale $(\mathrm{r}=.932, \mathrm{p}=.000)$ show that scores at time one and time two were highly correlated. Correlations for the FACT-Ntx were slightly lower $(r=.828, \mathrm{p}=.000)$ than for either scale of the CIPNAT.

Cronbach's alpha for the total CIPNAT, the symptom experience scale and its subscales, the interference scale and its subscales all demonstrated internal consistency reliability of the CIPNAT (Table 12). Item to total correlations ranged from .249-.688 (Table 13). Cronbach's alpha coefficients are dependent on test length as well as item variance (Waltz, Strickland, \& Lenz, 2005). Standardized alpha values can be adjusted using the Spearman-Brown formula to allow for comparison of independent alpha coefficients between scales of different lengths (Alsawalmeh \& Feldt, 1999). The alpha coefficient for the CIPNAT, adjusted using the Spearman-Brown formula, is estimated at .813 if it were reduced to an 11 item scale. Similarly, if the FACT/GOG-Ntx were lengthened to include 55 items, the estimated alpha is .948. Differences between these values were not significant indicating that the CIPNAT and the FACT/GOG-Ntx have comparable internal consistency reliability.

Table 12. Cronbach's alpha for CIPNAT and FACT-Ntx

\begin{tabular}{lcccc}
\hline Scale & $n$ & n of items & alpha & standardized alpha \\
\hline CIPNAT & 125 & 55 & .945 & .956 \\
CIPNAT symptom experience & 126 & 40 & .927 & .947 \\
Sensory subscale & 126 & 28 & .921 & .945 \\
Motor subscale & 127 & 12 & .890 & .924 \\
CIPNAT interference & 126 & 15 & .894 & .897 \\
Manual Dexterity subscale & 126 & 4 & .827 & .832 \\
General Activity subscale & 126 & 11 & .871 & .869 \\
FACT/GOG-Ntx & 127 & 11 & .784 & .784 \\
\hline
\end{tabular}




\section{Discussion}

The findings of this study support use of the CIPNAT as a reliable and valid selfreport tool for CIPN. It provides a comprehensive instrument for assessment of CIPN that addresses multidimensional elements including character, timing, intensity, distress and interference with activities. The CIPNAT is a useful tool for clinicians and researchers interested in the effects of CIPN on performance.

Validity. Validity evaluation including use of the contrasted groups approach, correlation with the FACT/GOG-Ntx and confirmatory factor analysis indicate that the CIPNAT is valid for assessing effects of neuropathy in cancer patients receiving chemotherapy. Differences between groups and correlation with the FACT/GOG-Ntx indicate that validity of the CIPNAT is strong.

The factor structure of the CIPNAT was different than hypothesized. It was expected that factor analysis of neuropathic symptoms would reveal two factors; either painful symptoms and non-painful symptoms or sensory symptoms and motor symptoms. The findings of this study support a two factor structure; sensory symptoms and motor symptoms. Examination of factor loadings in exploratory analysis favored selection of sensory symptoms and motor symptoms over painful symptoms and non-painful symptoms.

It also was expected that the interference subscale would consist of a single underlying construct. The findings of this study do not support a single factor solution but suggest that two factors, general activities and activities requiring manual dexterity, make up the interference scale. These findings are similar to those of Almadrones and 
colleagues (2004) who reported that general mobility and specific mobility were the underlying factors of the Peripheral Neuropathy Scale functional performance subscale. The findings highlight the importance of evaluating the impact of neuropathic symptoms on both specific activities as well as on general activities that can affect individual quality of life. The presence of a sensory symptom subscale and a motor symptom subscale support the idea that muscular symptoms are an important aspect of CIPN and should be included in routine assessment of CIPN.

Reliability. The results of this study indicate that the CIPNAT is a highly reliable tool for evaluation of CIPN. Test-retest reliability evaluation reveals very strong reliability. Internal consistency reliability also indicate that the CIPNAT is highly reliable and internally consistent, meaning answers to one item are a good indication of answers on other items (Waltz, Strickland, \& Lenz, 2005). Test-retest and internal consistency reliability were comparable to the FACT/GOG-Ntx.

Revisions to the CIPNAT. Validity and reliability information obtained in this phase III study guided further revisions to the CIPNAT. The items pertaining to "nerve pain" were revised to refer to "discomfort in the hands" and "discomfort in the feet" in an effort to help differentiate between neuropathic pain related to chemotherapy and pain related to other causes.

The amount of variance explained in nerve pain and cold sensitivity combined with low factor loadings for these variables suggest that these symptoms have other causes outside of CIPN (Kelloway, 1998) and may warrant further evaluation. 
Numbness and tingling in the hands will be evaluated on the same page and numbness and tingling in the feet will be evaluated on the same page to minimize confusion and redundancy. Numbness in the hands and tingling in the hands are highly correlated as are numbness in the feet and tingling in the feet. These correlations caused problems with goodness of fit in confirmatory factor analysis. Numbness and tingling often co-occur (Ocean \& Vahdat, 2004) and therefore patients may not be able to differentiate between the two. Feedback from patients during data collection supports this idea. Items on the CIPNAT were revised accordingly.

The item asking about interference with sexual activity will be eliminated because of low factor loadings as well as low item-total correlations and low occurrence. The item asking about injuries will be rephrased to ask about falls and will be included as part of further assessment of loss of balance. In addition, assessment of loss of balance will include asking patients if they use a walker or cane. Possible responses for this item will include "no", “yes, I used a cane or walker before I started chemo, or "yes, I started using a cane or walker since starting chemo". Both of these new items will be considered descriptive data and will not be included in scoring the CIPNAT.

Scoring of the CIPNAT. The resulting CIPNAT (v.1) is a 64 item self report tool with 51 items in the symptom experience scale and 13 items in the interference scale (Appendix F). Because a simplified scoring technique is desirable, scores on intensity, distress, and frequency will be summed to form a symptom experience scale score. Symptom experience scale scores may range from 0-240. Scores on the interference will be summed to form a total interference scale with a range of $0-130$. Total scores for the 
entire instrument range from 0-370. Higher scores correspond with more CIPN and more neuropathic interference with activities.

Limitations. Although the results of this study indicate that the CIPNAT is a reliable and valid self-report tool for CIPN, several limitations should be acknowledged. The sample size was not large, which may have had some impact on the results. Patients were predominantly from a large comprehensive cancer center in an urban area. Although this is a tertiary referral center, it serves a distinct geographic region, so the results may not be generalizable to other populations.

Confirmatory factor analysis using dichotomous variables is a controversial but widely implemented technique (Muthen, 1978). Tetrachoric correlation is an advanced technique used in factor analysis of dichotomous variables involving large sample sizes. Because this sample was small, it was not possible to use this technique. Our analysis may have been affected by not using tetrachoric correlations for factor analysis of the symptom experience subscale.

Implications for nursing. Assessment and management of side effects of chemotherapy, including CIPN, is predominantly a nursing responsibility. Unfortunately, nurses have not had access to good assessment tools for CIPN (ONS, 2005). This study provides an instrument that may be useful for clinical assessment of CIPN.

Effective prevention and treatment for CIPN is virtually non-existent. The best available treatment for CIPN is to stop chemotherapy or reduce the dose (ONS, 2005); however, this may lead to higher cancer morbidity and mortality. Sometimes less neurotoxic chemotherapy drugs are available but they are not always as effective in 
treating the underlying cancer. In all patients, particularly those with advanced or incurable disease, discussions about possible effects of neurotoxic chemotherapy, including impaired physical functioning and enjoyment of life should occur prior to initiation of chemotherapy. Evaluation of personal goals and lifestyle should guide treatment planning. Oncology nurses are integral to helping patients understand the potential neurotoxic side effects of chemotherapy and how these side effects may affect the patient's daily activities.

Nurses who are not directly involved with patients on chemotherapy may be unaware of symptoms of CIPN or how it potentially affects performance and quality of life. Attention to educating nurses about CIPN is critical because symptoms can last for many years after cessation of chemotherapy, making it a survivorship issue that must be addressed in research, education, and clinical practice.

Directions for future research. It is the goal of the author to develop and test a computerized version of the CIPNAT. This would help minimize missing data, allow for automatic scoring and reduce patient burden. Following development and validation of the computerized version of the CIPNAT, a computer based system for self-management of CIPN will be developed. Both the pen and pencil version and the computerized version of the CIPNAT will be used to test interventions aimed at decreasing neuropathic symptoms and/or minimizing neuropathic interference with performance status.

Conclusions. The results of this study confirm that the CIPNAT is a reliable and valid measure of CIPN in cancer patients receiving chemotherapy. The CIPNAT is more comprehensive than previously published instruments because it takes into account the 
multidimensional aspects of CIPN including character, intensity, timing, distress and interference with activities. An additional advantage of the CIPNAT is its strong data for reliability and validity. This study supports earlier research indicating that neurotoxic chemotherapy drugs produce both sensory and motor effects (Postma, et al. 2005; Huang, 2007). It also supports earlier research indicating that CIPN significantly interferes with patients' ability to perform specific tasks requiring manual dexterity as well as general activities including enjoyment of life (Almadrones, et al., 2005). 


\section{References}

University of Florida Shands Cancer Center (2007). from

http://www.ufscc.ufl.edu/Patient/content.aspx?section=ufscc\&id=23151

American Cancer Society (2008). Cancer Facts and Figures 2008. from http://www.cancer.org/downloads/STT/2008CAFFfinalsecured.pdf

Almadrones, L., McGuire, D. B., Walczak, J. R., Florio, C. M., \& Tian, C. (2004). Psychometric evaluation of two scales assessing functional status and peripheral neuropathy associated with chemotherapy for ovarian cancer: a gynecologic oncology group study. Oncology Nursing Forum, 31(3), 615-623.

Alsawalmeh, Y. M., Alsawalmeh, Y. M., \& Feldt, L. S. (1999). Testing the equality of two independent \&alpha; coefficients adjusted by the Spearman-Brown formula. Applied Psychological Measurement, 23(4), 363.

Alsawalmeh, Y. M., \& Feldt, L. S. (2000). A test of the equality of two related \&alpha; coefficients adjusted by the Spearman-Brown formula. Applied Psychological Measurement, 24(2), 163.

Backonja, M. M. (2003). Defining neuropathic pain. Anesthesia and Analgesia, 97(3), 785-790.

Badros, A., Goloubeva, O., Dalal, J. S., Can, I., Thompson, J., Rapoport, A. P., et al. (2007). Neurotoxicity of bortezomib therapy in multiple myeloma: A singlecenter experience and review of the literature. Cancer.

Bakitas, M. A. (2007). Background noise: The experience of chemotherapy-induced peripheral neuropathy. Nursing Research, 56(5), 323-331.

Berg, D. (2003). Oxaliplatin: a novel platinum analog with activity in colorectal cancer. Oncology Nursing Forum, 30(6), 957-966.

Calhoun, E. A., Welshman, E. E., Chang, C. H., Lurain, J. R., Fishman, D. A., Hunt, T. L., et al. (2003). Psychometric evaluation of the Functional Assessment of Cancer Therapy/Gynecologic Oncology Group-Neurotoxicity (Fact/GOG-Ntx) questionnaire for patients receiving systemic chemotherapy. International Journal of Gynecological Cancer, 13(6), 741-748. 
Cella, D., Peterman, A., Hudgens, S., Webster, K., \& Socinski, M. A. (2003). Measuring the side effects of taxane therapy in oncology: the functional assesment of cancer therapy-taxane (FACT-taxane). Cancer, 98(4), 822-831.

Chaudhry, V., Chaudhry, M., Crawford, T. O., Simmons-O'Brien, E., \& Griffin, J. W. (2003). Toxic neuropathy in patients with pre-existing neuropathy. Neurology, 60(2), 337-340.

Dunlap, B., \& Paice, J. A. (2006). Chemotherapy-induced peripheral neuropathy: A need for standardization in measurement. Journal of Supportive Oncology, 4(8), 398399.

Empl, M., Renaud, S., Erne, B., Fuhr, P., Straube, A., Schaeren-Wiemers, N., et al. (2001). TNF-alpha expression in painful and nonpainful neuropathies. Neurology, 56(10), 1371-1377.

Ferreira, K. A., Kimura, M., Teixeira, M. J., Mendoza, T. R., da Nobrega, J. C., Graziani, S. R., et al. (2008). Impact of cancer-related symptom synergisms on healthrelated quality of life and performance status. Journal of Pain and Symptom Management.

Gordon, A. N., Stringer, C. A., Matthews, C. M., Willis, D. L., \& Nemunaitis, J. (1997). Phase I dose escalation of paclitaxel in patients with advanced ovarian cancer receiving cisplatin: rapid development of neurotoxicity is dose-limiting. Journal of Clinical Oncology, 15(5), 1965-1973.

Grant, J. S., \& Davis, L. L. (1997). Selection and use of content experts for instrument development. Research in Nursing and Health, 20(3), 269-274.

Huang, H. Q., Brady, M. F., Cella, D., \& Fleming, G. (2007). Validation and reduction of FACT/GOG-Ntx subscale for platinum/paclitaxel-induced neurologic symptoms: a gynecologic oncology group study. International Journal of Gynecological Cancer, 17(2), 387-393.

Kasper, D., Fauci, A., Longo, D., Braunwald, E., Hauser, S., \& Jameson, J. (Eds.). (2005). Harrison's Principles of Internal Medicine (16th ed.): McGraw-Hill Companies, Inc.

Kelloway, E. (1998). Using LISREL for Structural Equation Modeling: A Researcher's Guide. Thousand Oaks: Sage Publications.

Kopec, J., Land, S., Cecchini, R., Ganz, P., Cella, D., Constantino, J., et al. (2006). Validation of a self-reported neurotoxicity scale in patients with operable colon cancer receiving oxaliplatin. Journal of Supportive Oncology, 4(8), W1-W8. 
Leidy, N. K. (1994). Functional status and the forward progress of merry-go-rounds: toward a coherent analytical framework. Nursing Research, 43(4), 196-202.

Lenz, E. R., Pugh, L. C., Milligan, R. A., Gift, A., \& Suppe, F. (1997). The middle-range theory of unpleasant symptoms: an update. Advances in Nursing Science, 19(3), 14-27.

Lenz, E. R., Suppe, F., Gift, A. G., Pugh, L. C., \& Milligan, R. A. (1995). Collaborative development of middle-range nursing theories: toward a theory of unpleasant symptoms. Advances in Nursing Science, 17(3), 1-13.

Lipton, R. B., Apfel, S. C., Dutcher, J. P., Rosenberg, R., Kaplan, J., Berger, A., et al. (1989). Taxol produces a predominantly sensory neuropathy. Neurology, 39(3), 368-373.

Mantyh, P. W. (2006). Cancer pain and its impact on diagnosis, survival and quality of life. Nature Reviews Neuroscience, 7(10), 797-809.

Merskey, H., \& Bogduk, N. (1994). Classifiication of chronic pain. Seattle: IASP Press.

Mulrooney, T. (2008). Cognitive impairment after breast cancer treatment. Clinical Journal of Oncology Nursing, 12(4), 678-680.

Muthen, B. (1978). Contributions to factor analysis of dichotomous variables. Psychometrika, 43(4), 551.

Ocean, A., \& Vahdat, L. (2004). Chemotherapy-induced peripheral neuropathy: pathogenesis and emerging therapies. Supportive Care in Cancer, 12(9), 619-625.

Oncology Nursing Society. (2005). Measuring oncology nursing -sensitive patient outcomes: evidence based summary for chemotherapy-induced peripheral neuropathy. Retrieved August 26, 2007, from http://www.ons.org/outcomes/measures/pdf/NeuropathySummary.pdf

Ostchega, Y., Donohue, M., \& Fox, N. (1988). High-dose cisplatin-related peripheral neuropathy. Cancer Nursing, 11(1), 23-32.

Pietrangeli, A., Leandri, M., Terzoli, E., Jandolo, B., \& Garufi, C. (2006). Persistence of high-dose oxaliplatin-induced neuropathy at long-term follow-up. European Neurology, 56(1), 13-16 
Portenoy, R., Thaler, H.., Kornblith, A., Lepore, J. Friedlander-Klar, H., Kiyasu, E., et al. (1994). The Memorial Symptom Assessment Scale: an instrument for the evaluation of symptom prevalence, characteristics and distress. European Journal of Cancer, 30A (9), 1326-1336.

Postma, T. J., Aaronson, N. K., Heimans, J. J., Muller, M. J., Hildebrand, J. G., Delattre, J. Y., et al. (2005). The development of an EORTC quality of life questionnaire to assess chemotherapy-induced peripheral neuropathy: the QLQ-CIPN20. European Journal of Cancer, 41(8), 1135-1139.

Quasthoff, S., \& Hartung, H. P. (2002). Chemotherapy-induced peripheral neuropathy. Journal of Neurology, 249(1), 9-17.

Smith, E. L., Whedon, M. B., \& Bookbinder, M. (2002). Quality improvement of painful peripheral neuropathy. Seminars in Oncology Nursing, 18(1), 36-43.

Sweeney, C. W. (2002). Understanding peripheral neuropathy in patients with cancer: background and patient assessment. Clinical Journey of Oncology Nursing, 6(3), 163-166.

Uceyler, N., Rogausch, J., Toyka, K., \& Sommer, C. (2007). Differential expression of cytokines in painful and painless neuropathies. Neurology, 69(1), $42-49$.

Visovsky, C. (2003). Chemotherapy-induced peripheral neuropathy. Cancer Investigation, 21(3), 439-451.

Waltz, C., Strickland, O., \& Lenz, E. (2005). Measurement in Nursing and Health Research (3rd ed.). New York: Springer Publishing Company.

Wickham, R. (2007). Chemotherapy-induced peripheral neuropathy: a review and implications for oncology nursing practice. Clinical Journal of Oncology Nursing, 11(3), 361-376.

Wilkes, G. (2007). Peripheral neuropathy related to chemotherapy. Seminars in Oncology Nursing, 23(3), 162-173.

Wynd, C. A., Schmidt, B., \& Schaefer, M. A. (2003). Two quantitative approaches for estimating content validity. Western Journal of Nursing Research, 25(5), 508518. 
Appendices 


\section{Appendix A. FACT/GOG-Ntx Version 4.}

By circling one (1) number per line, please indicate how true each statement has been for you during the past 7 days.

\begin{tabular}{|c|c|c|c|c|c|c|}
\hline & & $\begin{array}{l}\text { Not at } \\
\text { all }\end{array}$ & $\begin{array}{c}\text { A } \\
\text { little } \\
\text { bit }\end{array}$ & $\begin{array}{l}\text { Some- } \\
\text { what }\end{array}$ & $\begin{array}{l}\text { Quite } \\
\text { a bit }\end{array}$ & $\begin{array}{l}\text { Very } \\
\text { much }\end{array}$ \\
\hline $\begin{array}{c}\mathrm{NTX} \\
1\end{array}$ & $\begin{array}{l}\text { I have numbness or tingling in my } \\
\text { hands }\end{array}$ & 0 & 1 & 2 & 3 & 4 \\
\hline $\begin{array}{c}\mathrm{NTX} \\
2\end{array}$ & 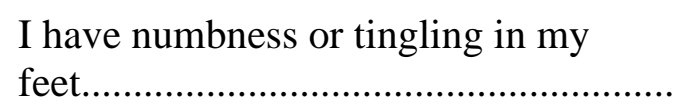 & 0 & 1 & 2 & 3 & 4 \\
\hline$\underset{3}{\mathrm{NTX}}$ & I feel discomfort in my hands ................ & 0 & 1 & 2 & 3 & 4 \\
\hline$\underset{4}{\mathrm{NTX}}$ & I feel discomfort in my feet.................... & 0 & 1 & 2 & 3 & 4 \\
\hline $\begin{array}{c}\mathrm{NTX} \\
5\end{array}$ & I have joint pain or muscle cramps ....... & 0 & 1 & 2 & 3 & 4 \\
\hline $\begin{array}{l}\mathrm{HI} \\
12\end{array}$ & I feel weak all over................................ & 0 & 1 & 2 & 3 & 4 \\
\hline$\underset{6}{\operatorname{NTX}}$ & 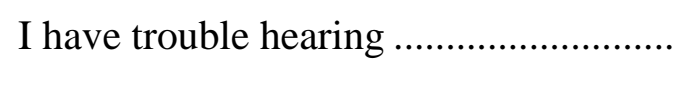 & 0 & 1 & 2 & 3 & 4 \\
\hline$\underset{7}{\mathrm{NTX}}$ & I get a ringing or buzzing in my ears .... & 0 & 1 & 2 & 3 & 4 \\
\hline$\underset{8}{\mathrm{NTX}}$ & I have trouble buttoning buttons ............ & 0 & 1 & 2 & 3 & 4 \\
\hline$\underset{9}{\mathrm{NTX}}$ & 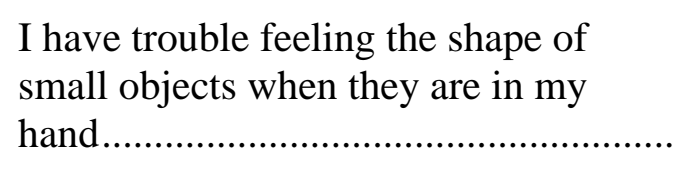 & 0 & 1 & 2 & 3 & 4 \\
\hline An6 & 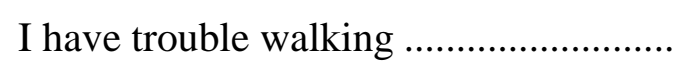 & 0 & 1 & 2 & 3 & 4 \\
\hline
\end{tabular}




\section{Appendix B. CIPNAT}

\section{Chemotherapy Induced Peripheral Neuropathy Assessment Tool (CIPNAT)}

Chemotherapy induced peripheral neuropathy occurs with certain types of chemotherapy that affect the nervous system. The following questions are designed to help us learn more about any symptoms of chemotherapy induced peripheral neuropathy that you might be experiencing. We are particularly interested in learning about symptoms that you have developed since receiving chemotherapy.

For pages 1-9 first read the question at the top of the page. If you answer yes than answer the rest of the questions on the page. If you answer no, skip to the next page. If you had the symptom before starting chemotherapy and there has been no change, answer no and move to the next page.

For page 10-11, if you have answered yes to any of the questions on the previous pages then circle a number 0-10 that corresponds with how much the symptoms you reported are interfering with your ability to perform certain activities. If you do not normally participate in the activity listed, please respond by circling 0 . If you answered no to the first question on each previous page, you do not need to complete pages 10-11.

Please answer every question that applies to you.

If you have physical problems that prevent you from being able to write, someone will read the questions to you and ask for your responses. If it is easier for you, someone else on the research team or a family member or friend can ask you the questions on the survey. We have tried to make it as brief and easy to complete as possible. If you get tired please feel free to take a break and come back to it later.

The information you provide is invaluable to us. Thank you. 
Appendix B (continued)

1 A. Since starting chemotherapy, have you developed numbness in your hands ? (circle one)

a. No (go to the next page)

b. Yes (answer the questions below)

1 B. How much of the hands are numb? (circle one)
a. Fingertips only
c. Entire hands
b. Fingertips and fingers
d. Entire hands and portions of the arms

$1 \mathrm{C}$. At its worst, how severe is the numbness in the hands? (circle one)

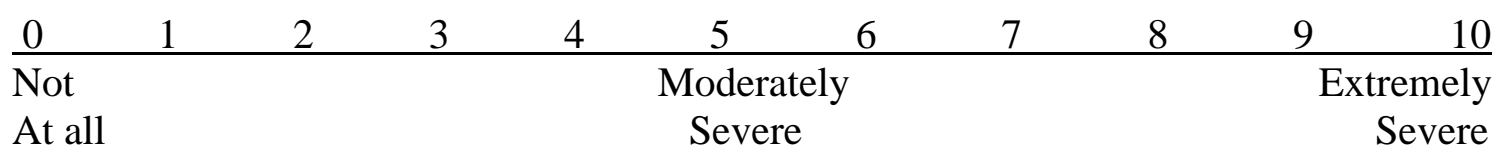

Severe

$1 \mathrm{D.}$ At its worst, how distressing is the numbness in the hands? (circle one)

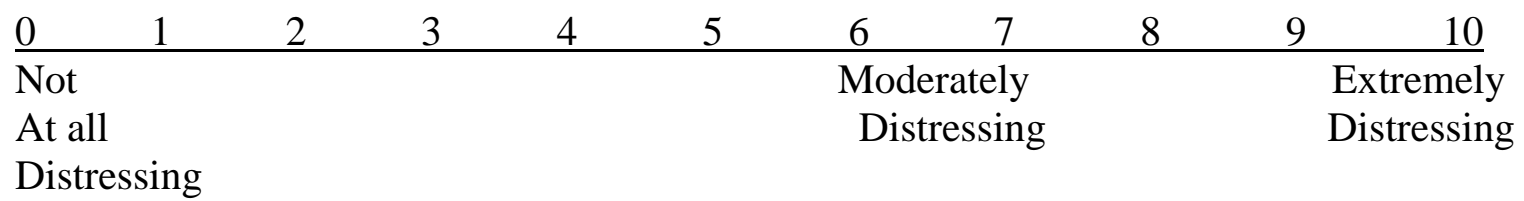

1 E. How often do you have numbness in the hands? (circle one)

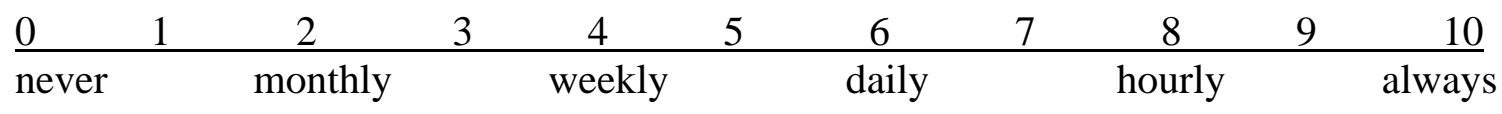

$1 \mathrm{~F}$. When is the numbness in the hands most severe? (circle as many as apply)
a. Mornings
d. Nights
b. Afternoons
e. After getting chemotherapy, for
c. Evenings days
f. Not applicable 
Appendix B (continued)

2 A. Since starting chemotherapy, have you developed numbness in the feet ? (circle one)

a. No (go to the next page)

b. Yes (answer the questions below)

2 B. How much of the foot is numb? (circle one)
a. Toes only
d. Entire foot
b. Toes and balls of feet
e. Entire foot/ parts of the leg
c. Toes, balls of feet, and sole of foot

$2 \mathrm{C}$. At its worst, how severe is the numbness in the feet? (circle one)

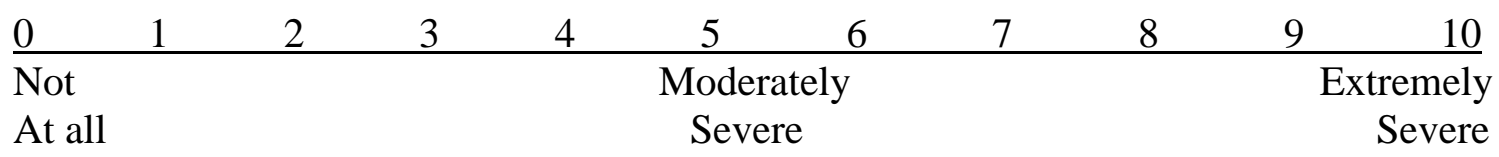

Severe

$2 \mathrm{D}$. At its worst, how distressing is the numbness in the feet? (circle one)

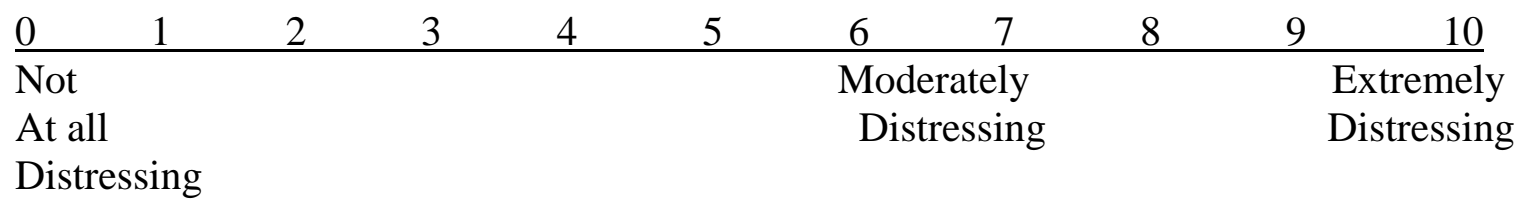

$2 \mathrm{E}$. How often do you have the numbness in the feet? (circle one)

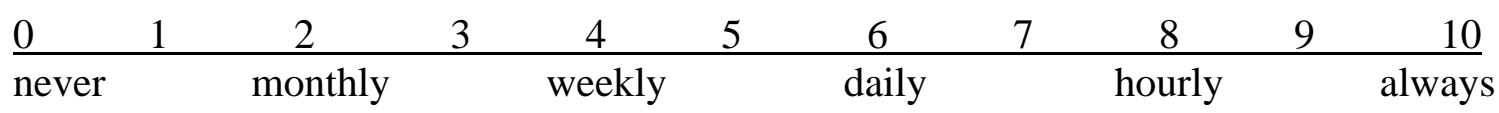

$2 \mathrm{~F}$. When is the numbness in the feet most severe? (circle as many as apply)
a. Mornings
d. Nights
b. Afternoons
e. After getting chemotherapy, for
c. Evenings days
f. Not applicable 
Appendix B (continued)

3 A. Since starting chemotherapy, have you developed tingling in the hands? (circle one)

a. No (go to the next page)

b. Yes (answer the questions below)

3 B. How much of the hands tingle? (circle one)
a. Fingertips only
c. Entire hands
b. Fingertips and fingers
d. Entire hands and portions of the arms

$3 \mathrm{C}$. At its worst, how severe is the tingling in the hands? (circle one)

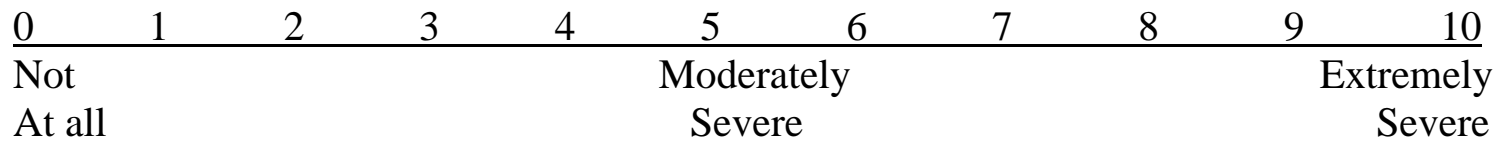

Severe

$3 \mathrm{D}$. At its worst, how distressing is the tingling in the hands? (circle one)

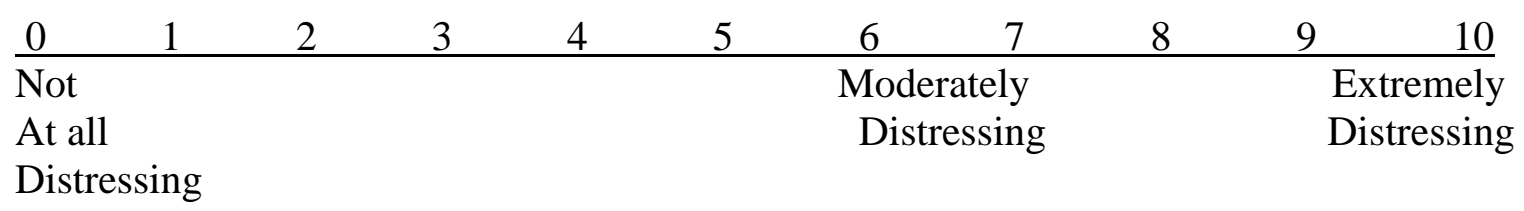

3 E. How often do you have tingling in the hands? (circle one)

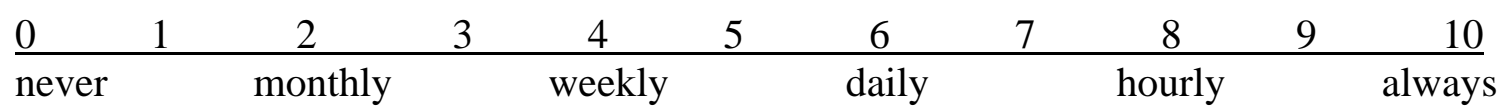

$3 \mathrm{~F}$. When is the tingling in the hands most severe? (circle as many as apply)
a. Mornings
d. Nights
b. Afternoons
e. After getting chemotherapy, for
c. Evenings days
f. Not applicable 
Appendix B (continued)

4 A. Since starting chemotherapy, have you developed tingling in the feet? (circle one)
a. No (go to the next page)
b. Yes (answer the questions below)

4 B. How much of the feet tingle? (circle one)
a. Toes only
d. Entire foot
b. Toes and balls of feet
e. Entire foot/ parts of the leg
c. Toes, balls of feet, and sole of foot

$4 \mathrm{C}$. At its worst, how severe is the tingling in the feet? (circle one)

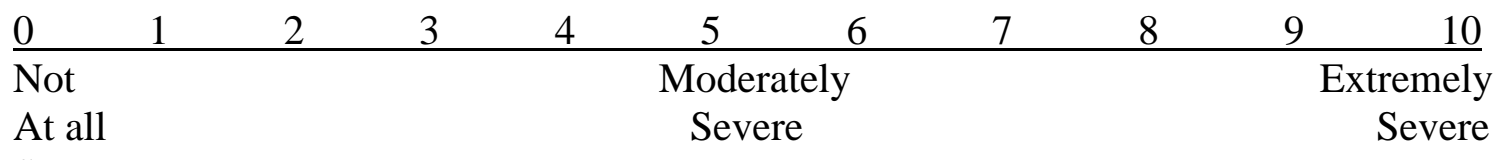

Severe

$4 \mathrm{D}$. At its worst, how distressing is the tingling in the feet? (circle one)

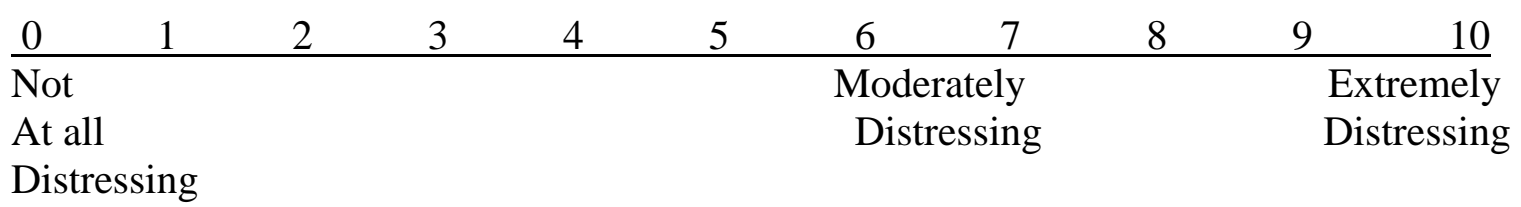

4 E. How often do you have the tingling in the feet? (circle one)

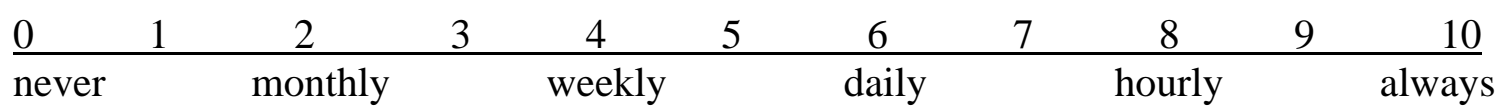

$4 \mathrm{~F}$. When is the tingling in the feet most severe? (circle as many as apply)
a. Mornings
d. Nights
b. Afternoons
e. After getting chemotherapy, for
c. Evenings days
f. Not applicable 
Appendix B (continued)

5 A. Since starting chemotherapy, have you developed sensitivity to cold temperatures? (circle one)
a. No (go to the next page)
b. Yes (answer the questions below)

5 B. What part(s) of your body are sensitive to cold? (circle as many as apply)
a. Hands
f. Abdomen
b. Arms
g. Throat
c. Feet
h. Jaw
d. Legs
i. Mouth
e. Back
j. Whole body

5 C. At its worst, how severe is the cold sensitivity? (circle one)

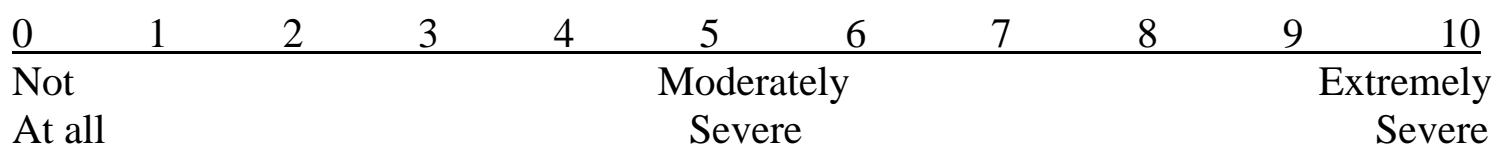

Severe

$5 \mathrm{D}$. At its worst, how distressing is the cold sensitivity? (circle one)

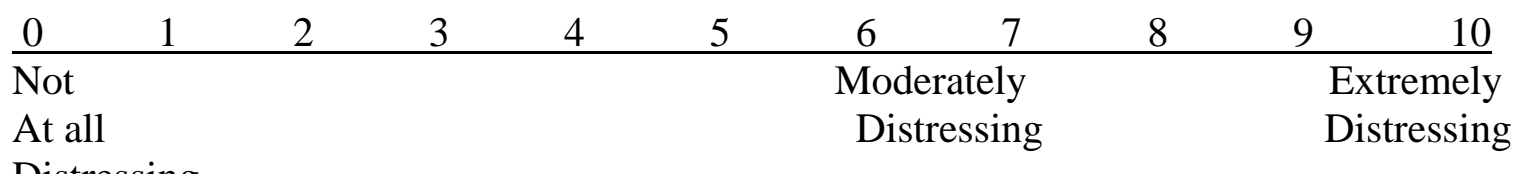

Distressing

5 E. How often do you have the cold sensitivity? (circle one)

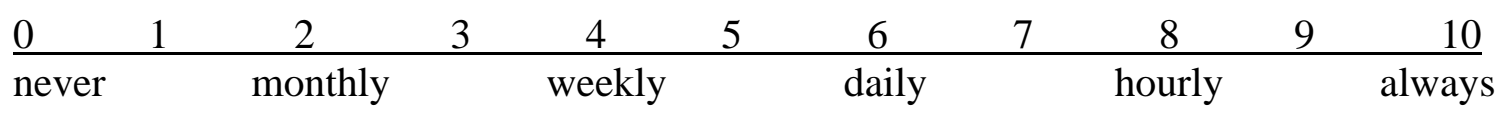

5 F. When is the cold sensitivity most severe? (circle as many as apply)
a. Mornings
d. Nights
b. Afternoons
e. After getting chemotherapy, for
c. Evenings days
f. Not applicable 
Appendix B (continued)

6 A. Since starting chemotherapy, have you had nerve pain (i.e. burning, shooting, stabbing, electric-like sensations)? (circle one)

a. No (go to the next page)

b. Yes (answer the questions below)

6B. What part(s) of your body have nerve pain? (circle as many as apply)
a. Hands
e. Jaw
b. Arms
f. Neck
c. Feet
g. Other
d. Legs

$6 \mathrm{C}$. At its worst, how severe is the nerve pain? (circle one)

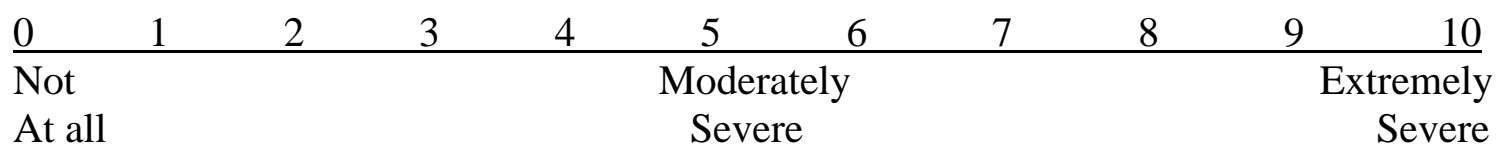

Severe

$6 \mathrm{D}$. At its worst, how distressing is the nerve pain? (circle one)

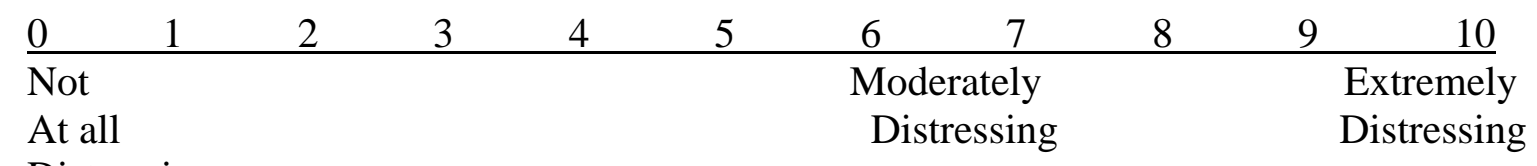

Distressing

6 E. How often do you have the nerve pain? (circle one)

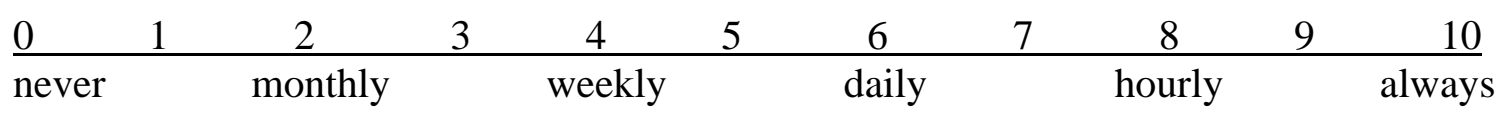

$6 \mathrm{~F}$. Circle the words that describe your nerve pain? (circle as many as apply)
a. Sharp
e. Stabbing
b. Shooting
f. Pins and needles
c. Burning
g. other
d. Electric-like

$6 \mathrm{G}$. When is the nerve pain most severe?(circle as many as apply)
a. Mornings
d. Nights
b. Afternoons
e. After getting chemotherapy, for
c. Evenings days
f. Not applicabl 
Appendix B (continued)

7 A. Since starting chemotherapy, have you developed muscle or joint aches? (circle one)
a. No (go to the next page)
b. Yes (answer the questions below)

7 B. What part(s) of your body ache? (circle as many as apply)
a. Muscles
e. Feet
b. Joints
f. Legs
c. Hands
g. Back
d. Arms
h. Whole body

7 C. At its worst, how severe is the aching? (circle one)

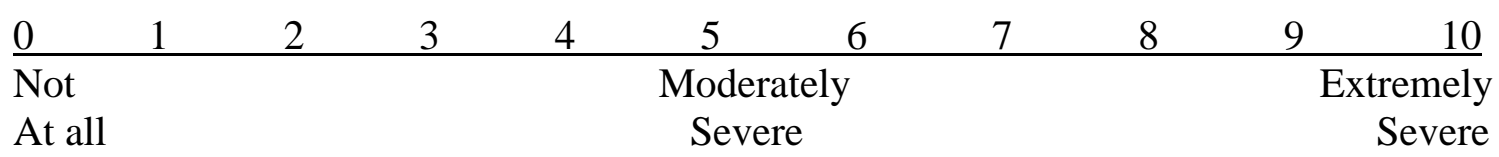

Severe

7 D. At its worst, how distressing is the aching? (circle one)

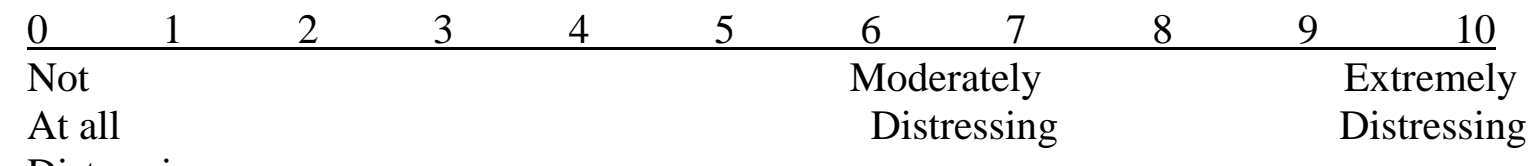

Distressing

7 E. How often do you have aching? (circle one)

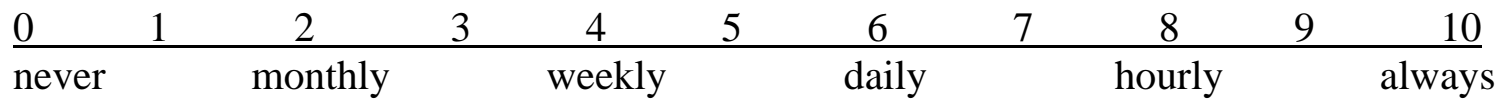

$7 \mathrm{~F}$. When is the aching most severe? (circle as many as apply)
a. Mornings
e. After getting chemotherapy, for
b. Afternoons days
c. Evenings
d. Nights
f. Not applicable 
Appendix B (continued)

8 A. Since starting chemotherapy, have your arms/hands or legs/feet felt weak? (circle one)

a. No (go to the next page)

b. Yes (answer the questions below)

8 B. What part(s) of your body have felt weak? (circle as many as apply)
a. Hands
c. Feet
b. Arms
d. Legs

$8 \mathrm{C}$. At its worst, how severe is the weakness? (circle one)

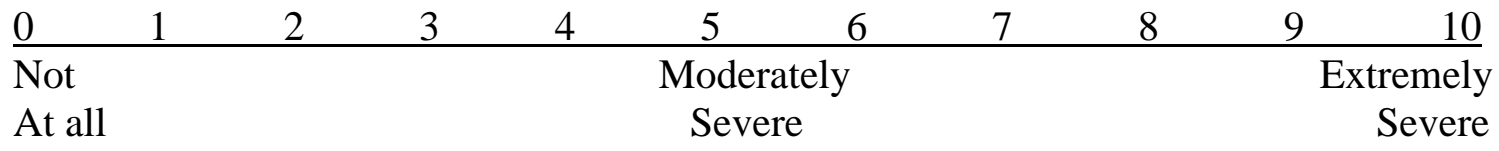

Severe

$8 \mathrm{D}$. At its worst, how distressing is the weakness? (circle one)

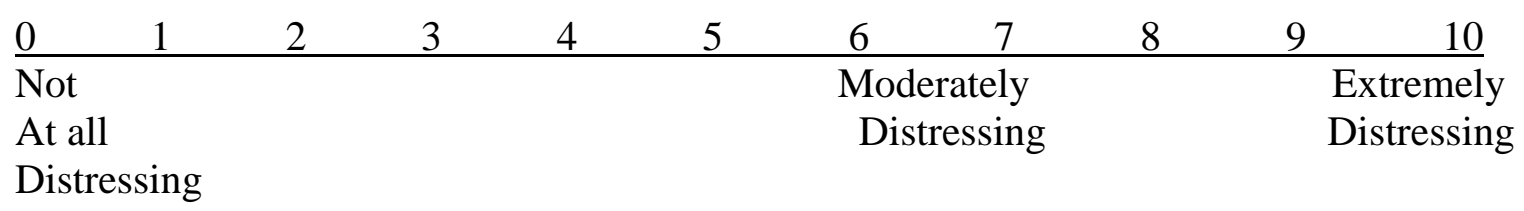

$8 \mathrm{E}$. How often do you have weakness in the arms/hands or legs/feet? (circle one)

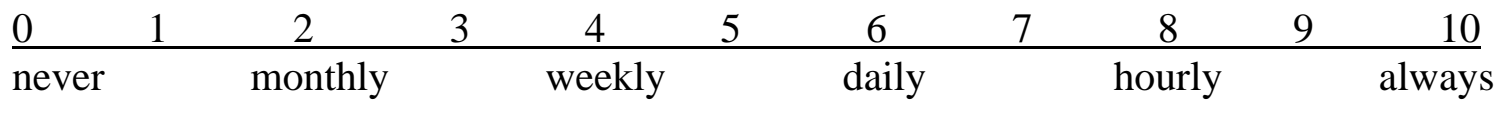

$8 \mathrm{~F}$. When is the weakness most severe? (circle as many as apply)
a. Mornings
d. Nights
b. Afternoons
e. After getting
c. Evenings chemotherapy,for days
f. Not applicable 
Appendix B (continued)

9 A. Since starting chemotherapy, have you had trouble with your balance? (circle one)
a. No (go to the next page)
b. Yes (answer the questions below)

9 B. At its worst, how severe is the trouble with your balance? (circle one)

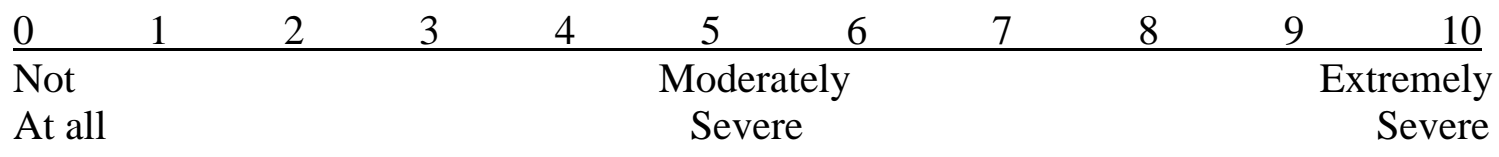

Severe

$9 \mathrm{C}$. At its worst, how distressing is the trouble with your balance? (circle one)

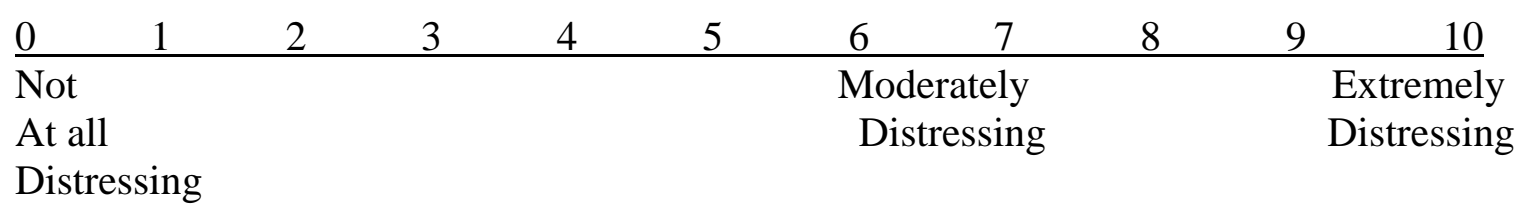

9 D. How often do you have trouble with your balance? (circle one)

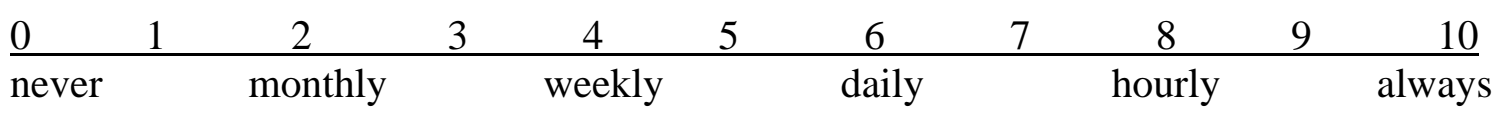

$9 \mathrm{E}$. When is the trouble with your balance most severe? (circle as many as apply)
a. Mornings
d. Nights
b. Afternoons
e. After getting chemotherapy, for
c. Evenings days
f. Not applicable 
Appendix B (continued)

If you answered yes to any of the previous symptoms, how much are your symptoms interfering with:

10 A. Dressing (buttoning, zipping, etc)

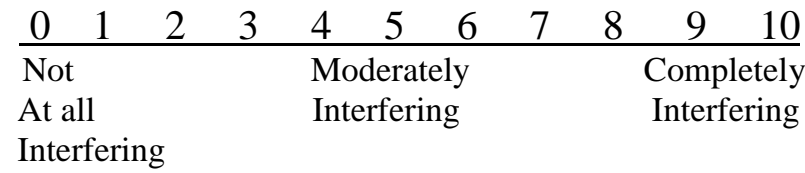

10 B. Walking

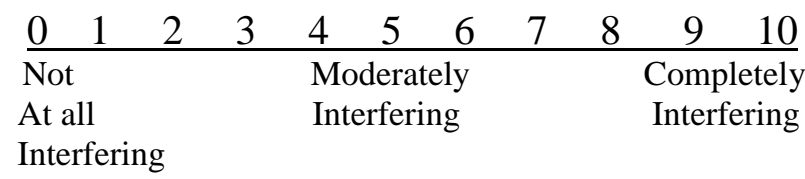

10 C. Picking up

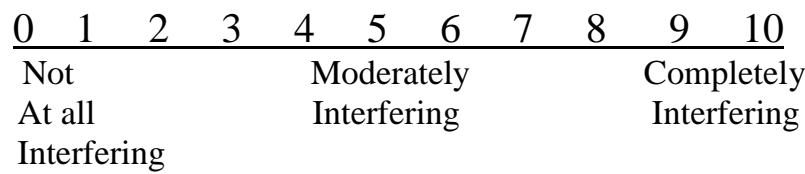

10 D. Holding onto objects

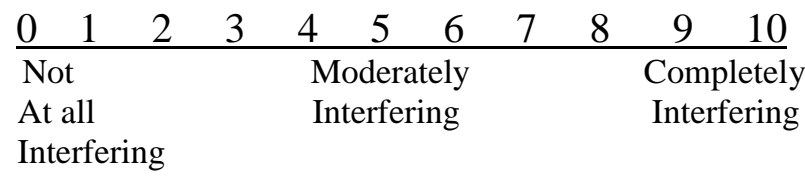

10 E. Driving

\begin{tabular}{lllllllllll}
0 & 1 & 2 & 3 & 4 & 5 & 6 & 7 & 8 & 9 & 10 \\
\hline Not & & & Moderately & & & Completely \\
At all & & & Interfering & & & Interfering
\end{tabular}

Interfering

10 F. Working

\begin{tabular}{lllllllllll}
0 & 1 & 2 & 3 & 4 & 5 & 6 & 7 & 8 & 9 & 10 \\
\hline Not & \multicolumn{4}{c}{ Moderately } & & & Completely \\
At all & & & Interfering & & & Interfering
\end{tabular}

Interfering

10 G. Participating in hobbies/ leisure activities

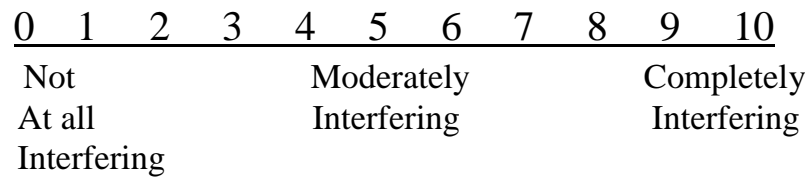

10 H. Exercising

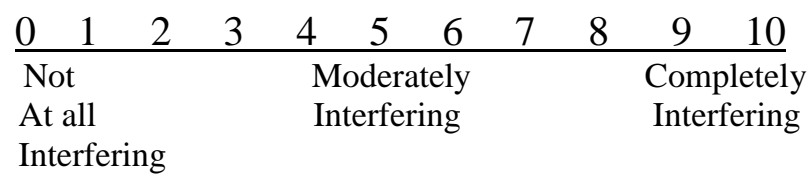


Appendix B (continued)

10 I. Sexual Activity

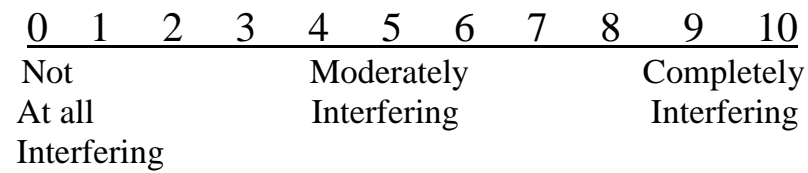

10 J. Sleeping

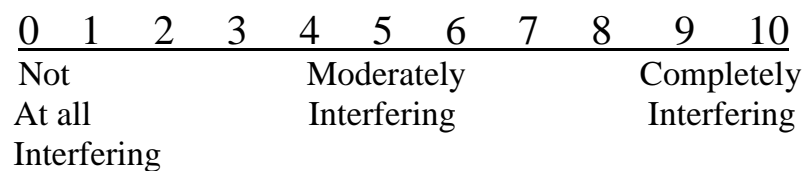

10 K. Relationships with other people

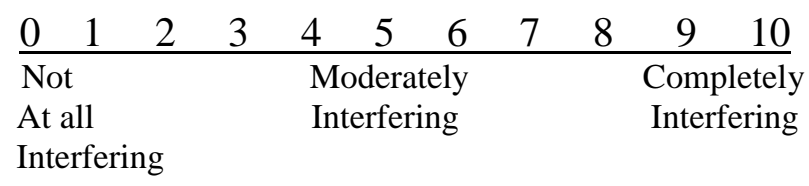

10 L. Writing

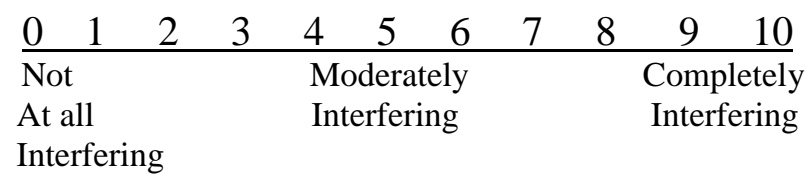

10 M. Usual household

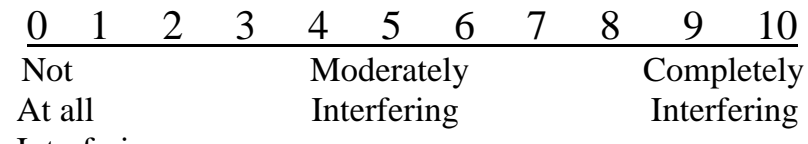

Interfering

$10 \mathrm{~N}$. Enjoyment of life

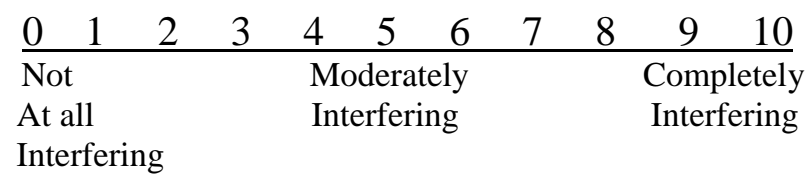

Have you had any bodily injuries (even minor) because of the symptoms you have reported as part of this survey (numbness, weakness, problems with balance, etc)?

No Yes, describe

Thank you for your time 
Appendix C. Demographic Data Form

Page one to be completed by participant.

1. What is your current age?

2. Which gender are you (circle one)? Male Female

3. What is your marital status (check one)?

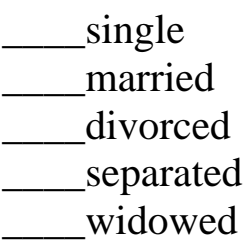

4. What is your annual household income (check one)?

less than 24,999 dollars per year

25,000-49,999 dollars per year 50,000-74,999 dollars per year more than 75,000 dollars per year prefer not to answer

5. How many years of formal education have you completed?

Example: $12=$ high school graduate $14=$ associate degree or technical school $16=$ baccalaureate degree

6. What is your race or ethnicity (check all that apply)

American Indian/Alaskan Native

Asian

African American

Hispanic

Native Hawaiian/Pacific Islander

White/Caucasian

other or unknown

7. What is your current employment status (check one):

full time employee

part time employee

on leave of absence

retired

disabled

full time student 
Appendix C (continued)

To be completed by researcher:

8. Type of cancer (circle one) : breast lung colon

9. Stage of cancer

10. Type of chemotherapy receiving (check all that apply)

_paclitaxel docetaxel cisplatin oxaliplatin

11. Cycle Number

12. Cumulative doses

$\begin{array}{ll}\text { Drug___ dose__ } & \mathrm{mg} / \mathrm{m}^{2} \\ \text { Drug__ose } & \mathrm{mg} / \mathrm{m}^{2}\end{array}$


Appendix D. Moffitt SRC Approval Letter

Wednesday, July 09, 2008

Cindy Tofthagen, MS, ARNP, AOCNP

Moffitt Cancer Center

University of South Florida

12902 Magnolia Drive

Tampa, FL 33612

Dear Dr. McMillan:

The Behavioral Scientific Review Committee (SRC) has approved your research protocol dated 7/2/2008 entitled, "Psychometric Evaluation of the Chemotherapy Induced Peripheral Neuropathy Assessment Tool" (MCC 15639) for use at the Moffitt Cancer Center under the Center's expedited review policy pending approval of the Institutional Review Board (IRB) and satisfaction of institutional operational and financial review requirements. Please be aware that after you receive IRB approval, you must submit a copy of the IRB approval letter to the Protocol Review and Monitoring System and request study activation before you commence any study activities. The Protocol Review and Monitoring System will ensure that all applicable institutional reviews have been completed. You will then be issued an activation letter. Upon receipt of the activation letter, you will be able to conduct your study.

It is your responsibility to ensure that all Moffitt staff (Nursing, Pharmacy, Data Management, etc.) is informed and aware of the details of the project. The Scientific Review Committee encourages the use of in-services for those projects which are complex or require special attention.

All changes made to protocols approved by the SRC must be submitted to the Protocol Review and Monitoring System. Substantial changes (i.e., scientific changes, therapy changes or eligibility changes) require SRC review and approval. 
Appendix D (continued)

If this project is not being managed by the Protocol Review and Monitoring System, then it is your responsibility to follow through will all requirements of the IRB as listed on page 5 of the "Application for Approval of Investigation Involving the Use of Human Subjects USF Health Sciences Center IRB". After the study is approved, the IRB requires submission of a continuing review report annually. Please forward a copy of this report to the Protocol Review and Monitoring System.

Oncore is the Cancer Center's mechanism for required submission and review of materials requiring IRB review as well as items requiring review by the Scientific Review and Protocol Monitoring Committees. If you are not currently reporting the necessary research activities, such as patient accrual, changes in procedure, adverse events, and continuing reviews in Oncore, please contact Jeryl Madden, Oncore Coordinator, at 745-6964 for direction.

Sincerely,

Paul Jacobsen, $\mathrm{PhD}$

Chair, Behavioral Subcommittee

Scientific Review Committee 
Appendix E. Letter of approval, private practice.

July 10, 2008

To Whom it May Concern,

I have reviewed the research proposal by Cindy Tofthagen entitled Psychometric Evaluation of the Chemotherapy Induced Peripheral Neuropathy Assessment Tool. I find the research appropriate for my oncology patients and give the investigator permission to conduct research in this office. Appropriate resources are available for this research to be conducted, including a private room for consenting and interviewing patients. Based on the minimal risks associated with this research, adequate provisions are available to handle any unanticipated events. Thank you for your time and attention to this matter.

Ron D. Schiff, M.D., Ph.D.

Egberto Zayas, M.D. 
Appendix F: CIPNAT (Version 1)

\section{Chemotherapy Induced Peripheral Neuropathy Assessment Tool (CIPNAT) Version 1}

Chemotherapy induced peripheral neuropathy occurs with certain types of chemotherapy that affect the nervous system. The following questions are designed to help us learn more about any symptoms of chemotherapy induced peripheral neuropathy that you might be experiencing. We are particularly interested in learning about symptoms that you have developed since receiving chemotherapy.

For pages 1-8 first read the question at the top of the page. If you answer yes than answer the rest of the questions on the page. If you answer no, skip to the next page. If you had the symptom before starting chemotherapy and there has been no change, answer no and move to the next page.

For page 9-10, if you have answered yes to any of the questions on the previous pages then circle a number 0-10 that corresponds with how much the symptoms you reported are interfering with your ability to perform certain activities. If you do not normally participate in the activity listed, please respond by circling 0 . If you answered no to the first question on each previous page, you do not need to complete pages 9-10.

Please answer every question that applies to you.

If you have physical problems that prevent you from being able to write, someone will read the questions to you and ask for your responses. If it is easier for you, someone else on the research team or a family member or friend can ask you the questions on the survey. We have tried to make it as brief and easy to complete as possible. If you get tired please feel free to take a break and come back to it later.

The information you provide is invaluable to us. Thank you. 
Appendix F(continued)

1A. Since starting chemotherapy, have you developed numbness or tingling in your hands? (circle one)

a. No (go to the next page)

b. Yes (answer the questions below)

1B. Which do you have (circle one)?
a. Numbness
b. Tingling
c. Both numbness and tingling

1C. How much of the hands have numbness or tingling? (circle one)
a. Fingertips only
b. Fingertips and fingers
c. Entire hands
d. Entire hands and portions of the arm

$1 \mathrm{D}$. At its worst, how severe is the numbness or tingling in the hands? (circle one)

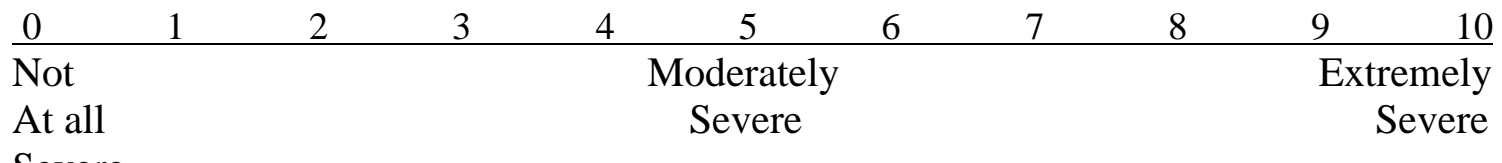

Severe

1 E. At its worst, how distressing is the numbness or tingling in the hands? (circle one)

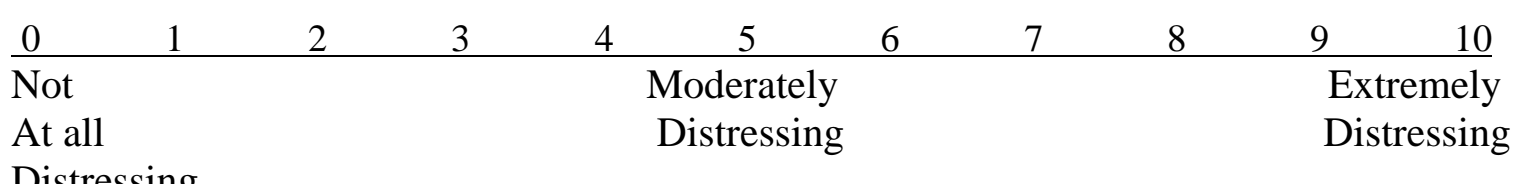

1 F. How often do you have numbness or tingling in the hands? (circle one)

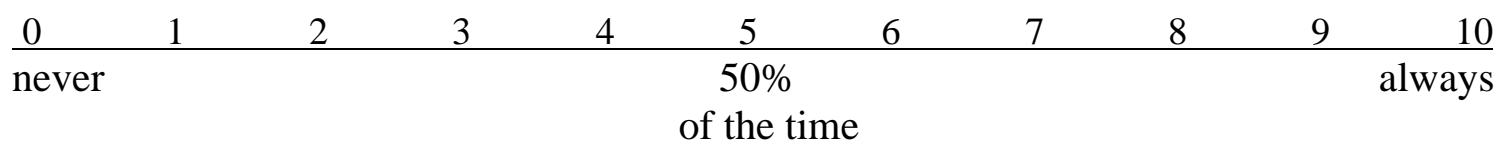

$1 \mathrm{G}$. Is the numbness or tingling in your hands worse after getting chemo ? (circle one)
a. No
b. Yes, for how many days 
Appendix F(continued)

2A. Since starting chemotherapy, have you developed numbness or tingling in your feet? (circle one)

a. No (go to the next page)

b. Yes (answer the questions below)

2B. Which do you have (circle one)?

a. Numbness

b. Tingling

c. Both numbness and tingling

2 C. How much of the feet tingle? (circle one)

a. Toes only

b. Toes and balls of feet

c. Entire foot

d. Entire foot/ parts of the leg

2 D. At its worst, how severe is the numbness/tingling in the hands? (circle one)

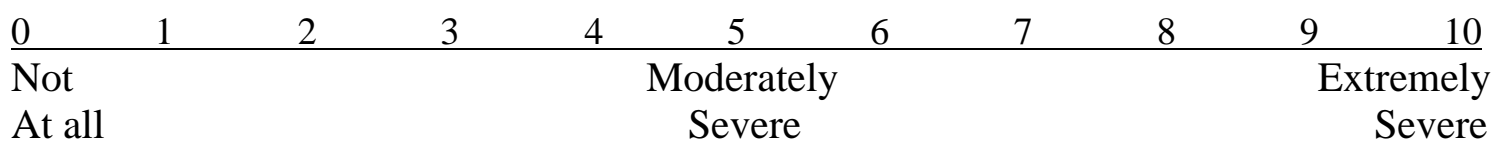

Severe

2 E. At its worst, how distressing is the numbness in the hands? (circle one)

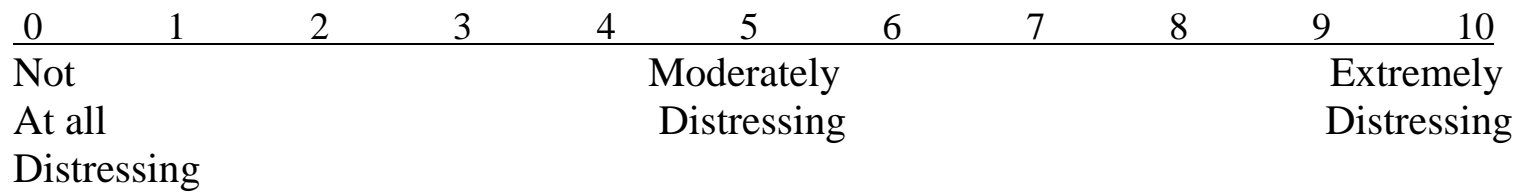

2 F. How often do you have numbness in the hands? (circle one)

$\begin{array}{lllllllllr}0 & 1 & 2 & 3 & 4 & 5 & 6 & 7 & 8 & 9\end{array}$

$2 \mathrm{G}$. Is the numbness or tingling in your hands worse after getting chemo? (circle one)
a. No
b. Yes, for how many days 
Appendix F(continued)

3 A. Since starting chemotherapy, have you had discomfort in your hands (circle one)?

a. No (go to the next page)

b. Yes (answer the questions below)

3 B. At its worst, how severe is the discomfort in your hands? (circle one)

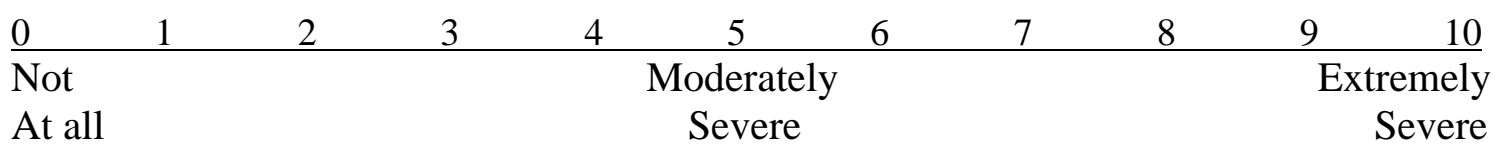

Severe

3 C. At its worst, how distressing is the discomfort in your hands? (circle one)

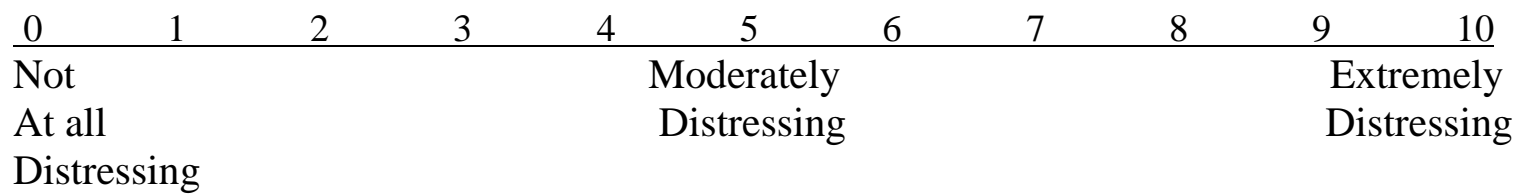

3 D. How often do you have the discomfort in your hands? (circle one)

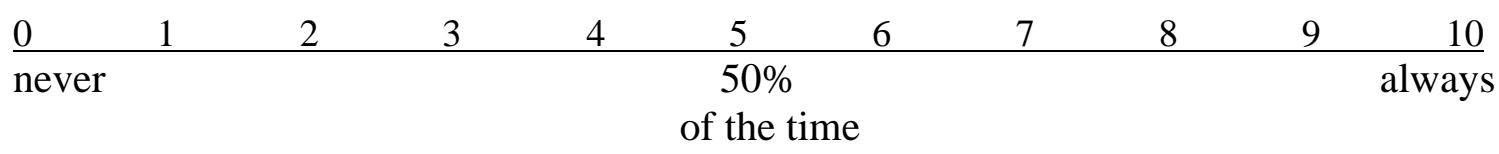

3E. Circle the words that describe the discomfort in your hands? (circle as many as apply)
a. Sharp
b. Shooting
c. Burning
d. Electric-like
e. Stabbing
f. Pins and needles
g. other

3 F. Is the discomfort in your hands worse after getting chemo? (circle one)
a. No
b. Yes, for how many days 
Appendix F(continued)

4 A. Since starting chemotherapy, have you had discomfort in your feet (circle one)?

a. No (go to the next page)

b. Yes (answer the questions below)

4 B. At its worst, how severe is the discomfort in your feet? (circle one)

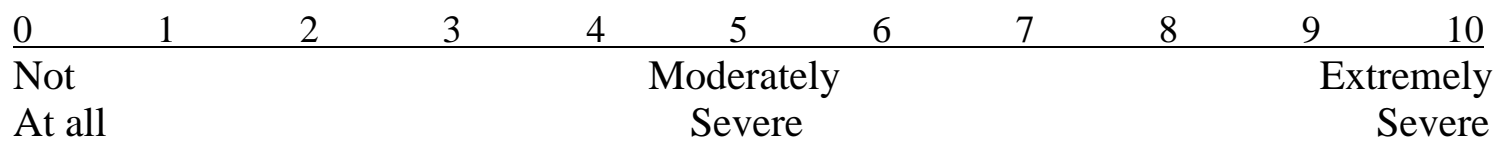

Severe

4 C. At its worst, how distressing is the discomfort in your feet? (circle one)

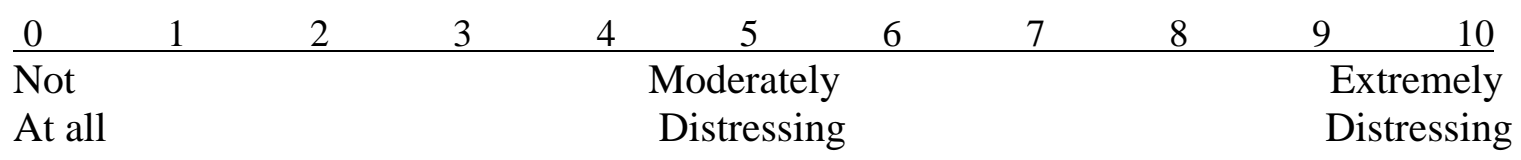

Distressing

4 D. How often do you have the discomfort in your feet? (circle one)

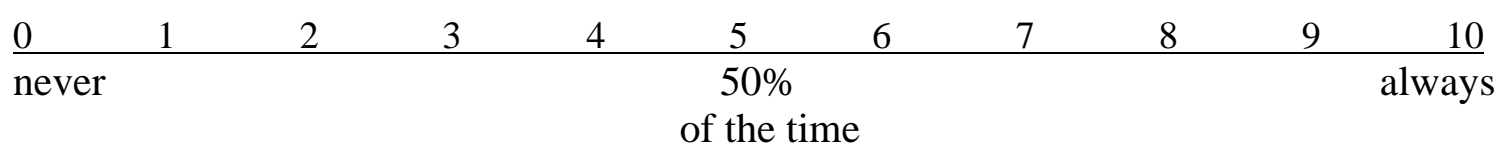

4 E. Circle the words that describe the discomfort in your feet? (circle as many as apply)
a. Sharp
b. Shooting
c. Burning
d. Electric-like
e. Stabbing
f. Pins and needles
g. other

$4 \mathrm{~F}$. Is the discomfort in your feet worse after getting chemo? (circle one)
a. No
b. Yes, for how many days? 
Appendix F(continued)

5 A. Since starting chemotherapy, have you developed sensitivity to cold temperatures? (circle one)
a. No (go to the next page)
b. Yes (answer the questions below)

5 B. What part(s) of your body are sensitive to cold? (circle as many as apply)
a. Hands
b. Feet
c. Throat
d. Jaw
e. Mouth
f. Whole body

5 C. At its worst, how severe is the cold sensitivity? (circle one)

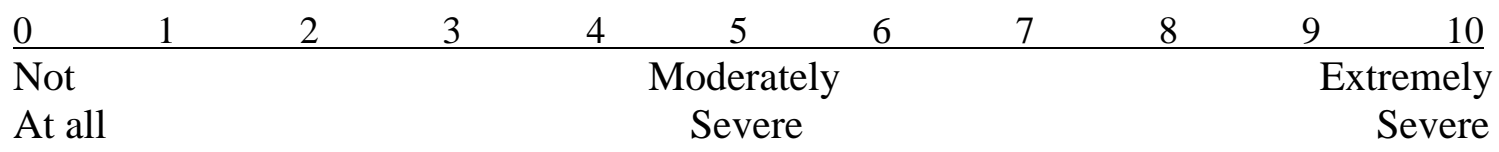

Severe

5 D. At its worst, how distressing is the cold sensitivity? (circle one)

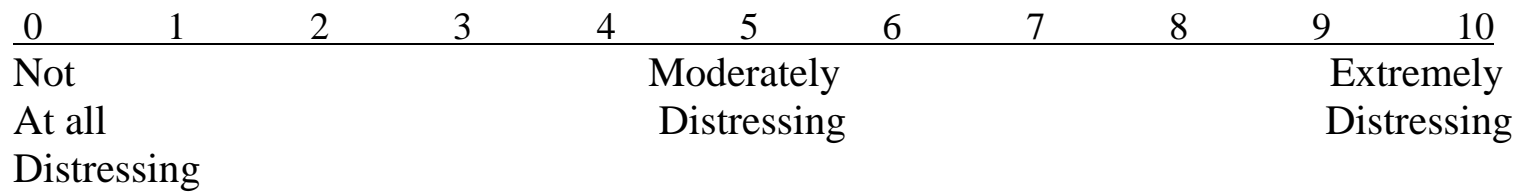

5 E. How often do you have the cold sensitivity? (circle one)

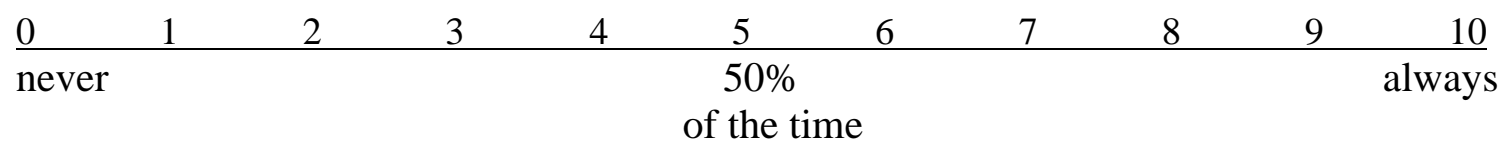

5 F. Is the cold sensitivity worse after getting chemo? (circle one)

a. No

b. Yes, for how many days 
Appendix F(continued)

6A. Since starting chemotherapy, have you developed muscle or joint aches? (circle one)

a. No (go to the next page)

b. Yes (answer the questions below)

6B. What part(s) of your body ache? (circle as many as apply)
a. Muscles
b. Joints
c. Both muscles and joints

$6 \mathrm{C}$. At its worst, how severe is the aching? (circle one)

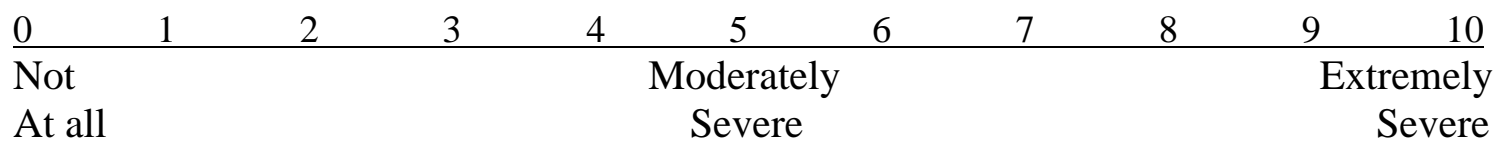

Severe

$6 \mathrm{D}$. At its worst, how distressing is the aching? (circle one)

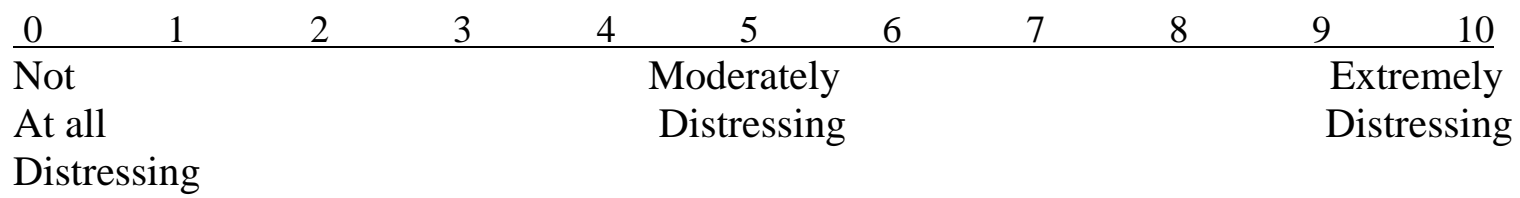

6 E. How often do you have aching? (circle one)

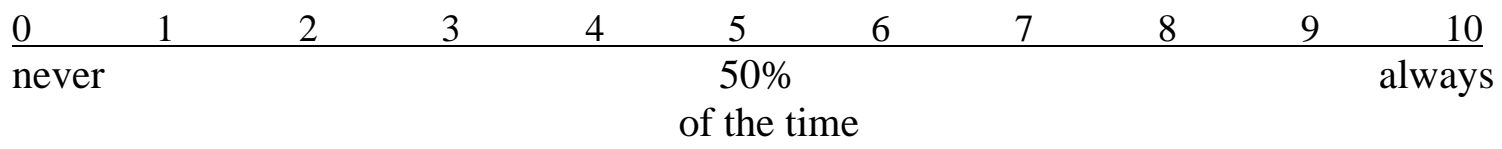

$6 \mathrm{~F}$. Is the aching worse after getting chemo? (circle one)
a. No
b. Yes, for how many days 
Appendix F(continued)

7A. Since starting chemotherapy, have your arms or legs felt weak? (circle one)

a. No (go to the next page)

b. Yes (answer the questions below)

7B. What part(s) of your body have felt weak? (circle as many as apply)
a. Arms
b. Legs
c. Both arms and legs

7 C. At its worst, how severe is the weakness in the arms or legs? (circle one)

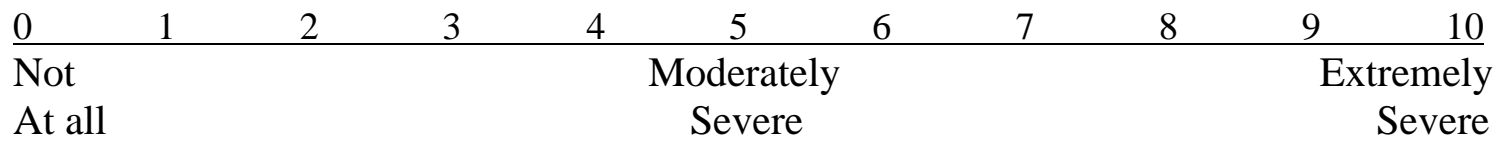

Severe

$7 \mathrm{D}$. At its worst, how distressing is the weakness in the arms or legs? (circle one)

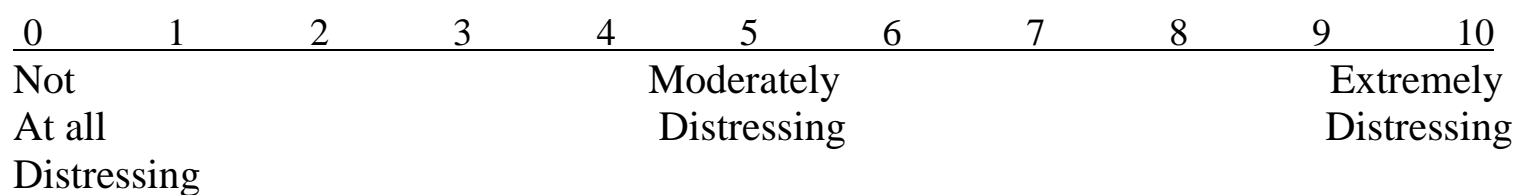

7 E. How often do you have weakness in the arms or legs? (circle one)

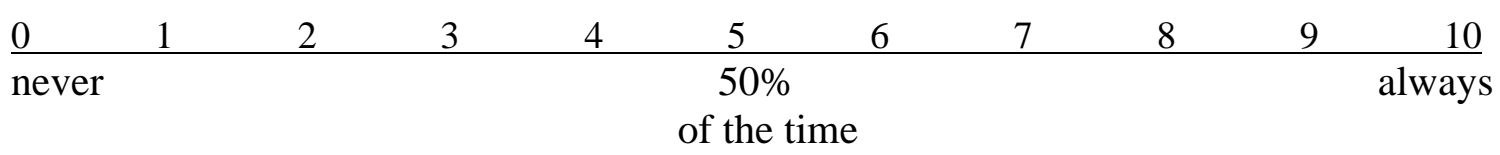

7 F. Is the weakness in the arms or legs worse after getting chemo? (circle one)
a. No
b. Yes, for how many days? 
Appendix F(continued)

8A. Since starting chemotherapy, have you had trouble with your balance? (circle one)

a. No (go to the next page)

b. Yes (answer the questions below)

$8 \mathrm{~B}$. At its worst, how severe is the trouble with your balance? (circle one)

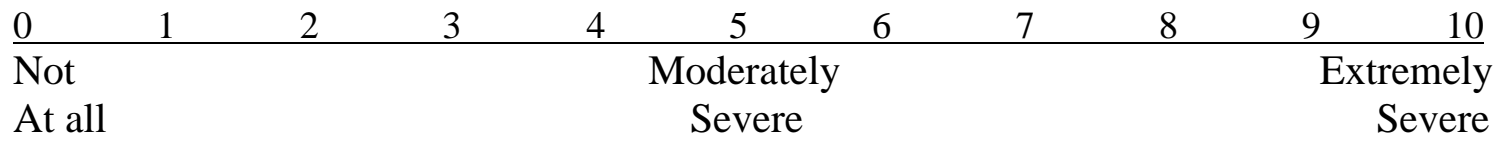

Severe

$8 \mathrm{C}$. At its worst, how distressing is the trouble with your balance? (circle one)

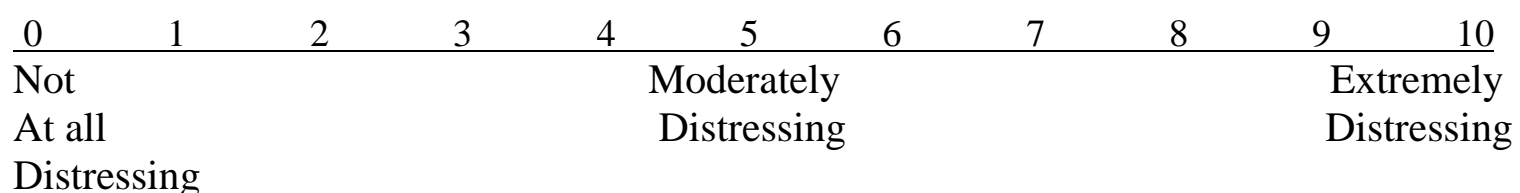

8 D. How often do you have trouble with your balance? (circle one)

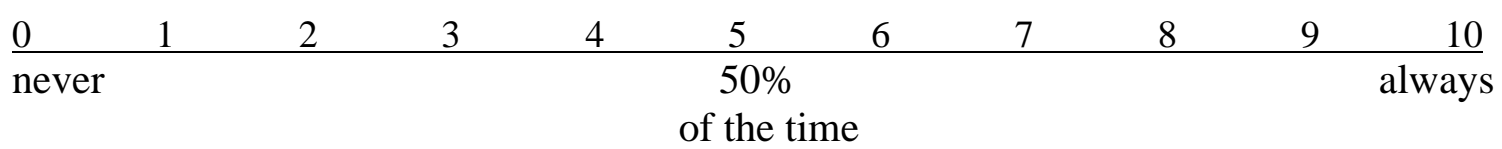

8 E. Have you had any falls since starting chemo?

a. No

b. Yes, describe

\section{F. Do you use a cane or walker?}

a. No

b. Yes, I used a cane or walker before I started chemo

c. Yes, I started using a cane or walker since starting chemo

8G. Is the loss of balance worse after getting chemo? (circle one)

a. No

b. Yes, for how many days 


\section{Appendix F(continued)}

If you answered yes to any of the previous symptoms, how much are your symptoms interfering with:

9 A. Dressing (buttoning, zipping, etc) \begin{tabular}{lllllllllllr}
0 & 1 & 2 & 3 & 4 & 5 & 6 & 7 & 8 & 9 & 10 \\
\hline Not & \multicolumn{1}{c}{ Moderately } & & Completely
\end{tabular}

At all Interfering Interfering

Interfering

9 B. Walking

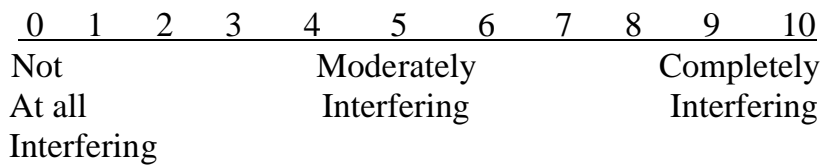

9 C. Picking up objects

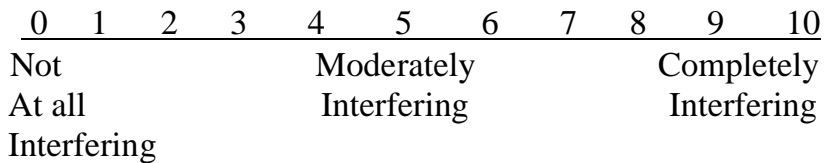

9 D. Holding onto objects

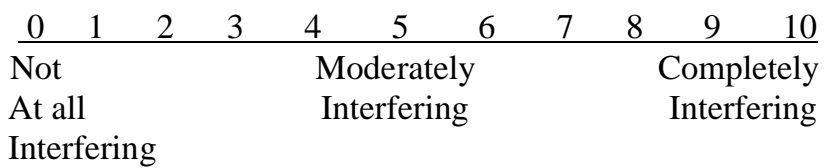

9 E. Driving

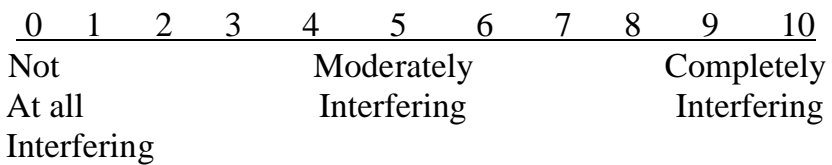

9 F. Working

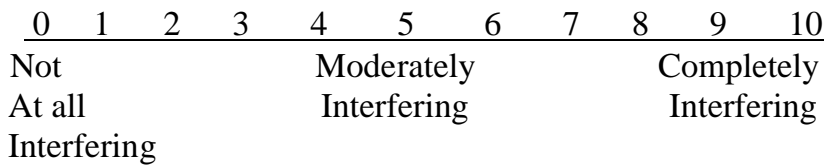

9 G. Participating in hobbies or leisure activities

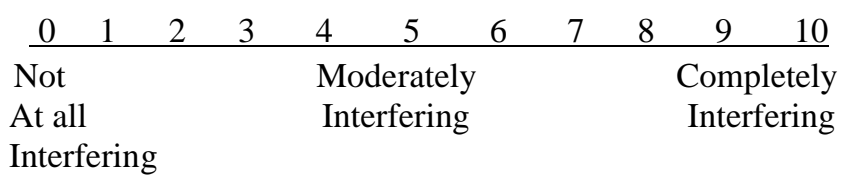

9 H. Exercising

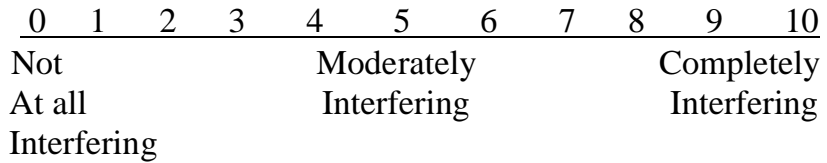

\section{I. Sleeping}

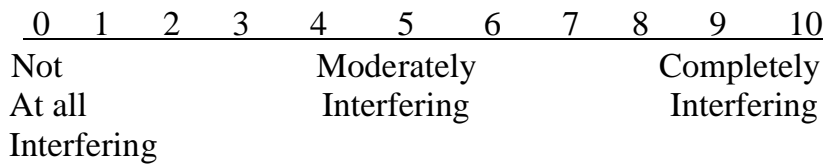




\section{Appendix F(continued)}

9 J. Relationships with other people

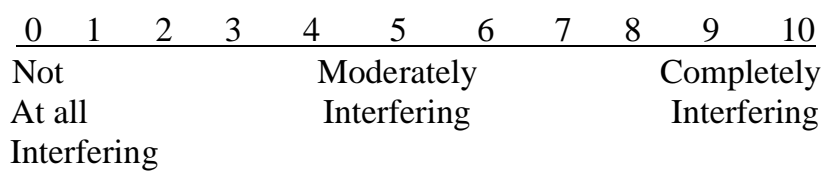

9 K. Writing

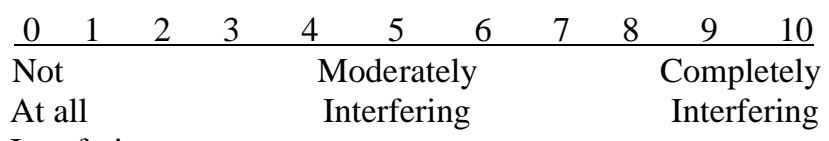

Interfering

9 L. Usual household chores

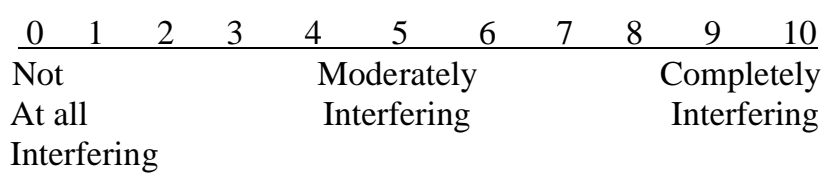

9 M. Enjoyment of life

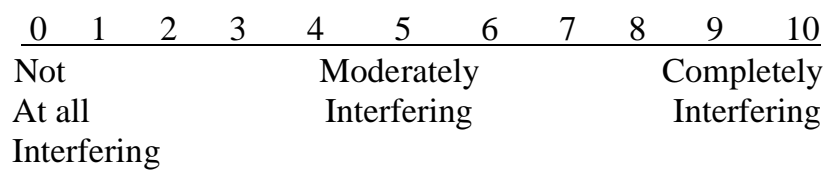




\begin{abstract}
About the Author
Cindy S. Tofthagen has over sixteen years of experience as a registered nurse specializing in oncology. She has extensive experience in chemotherapy and biotherapy as well as symptom management. Currently, she is an instructor at the University of South Florida College of Nursing, an Advanced Registered Nurse Practitioner for Ron D. Schiff, MD. She earned her Associate Degree in Nursing from Hillsborough Community College in 1992, her Bachelor of Science in Nursing from St. Petersburg College in 2004, and her Master of Science in Nursing with a concentration in oncology from the University of South Florida in 2006. Her research interests include survivorship issues, oncology symptom management, palliative care, and psychology of chronic illness. Her doctoral dissertation was funded by a scholarship from the American Cancer Society.
\end{abstract}


